\title{
A CLASS OF COMPACT POINCARÉ-EINSTEIN MANIFOLDS: PROPERTIES AND CONSTRUCTION
}

\author{
A. ROD GOVER AND FELIPE LEITNER
}

\begin{abstract}
We develop a geometric and explicit construction principle that generates classes of Poincaré-Einstein manifolds, and more generally almost Einstein manifolds. Almost Einstein manifolds satisfy a generalisation of the Einstein condition; they are Einstein on an open dense subspace and, in general, have a conformal scale singularity set that is a conformal infinity for the Einstein metric. In particular, the construction may be applied to yield families of compact Poincaré-Einstein manifolds, as well as classes of almost Einstein manifolds that are compact without boundary. We obtain classification results which show that the construction essentially exhausts a class of almost Einstein (and Poincaré-Einstein) manifold. We develop the general theory of fixed conformal structures admitting multiple compatible almost Einstein structures. We also show that, in a class of cases, these are canonically related to a family of constant mean curvature totally umbillic embedded hypersurfaces.
\end{abstract}

\section{INTRODUCTION}

On a compact manifold $M^{d}$ with boundary, a metric $g^{o}$ on the interior $M^{o}$ of $M$ is said to be conformally compact if $g^{o}=s^{-2} g$ where $g$ is nondegenerate up to the boundary $\partial M$ and $s$ is a defining function for $\partial M$. If also $g^{o}$ is Einstein then the structure is said to be Poincaré-Einstein (PE). The term asymptotically hyperbolic Einstein is also used for these structures which were introduced by Fefferman and Graham in [24] in connection with their ambient metric, and as a tool for studying the conformal geometry of the boundary. The relationship between that conformal structure and the geometry of the Riemannian interior has recently been studied intensively using spectral and scattering tools [25, 41, 42, 47, 56], and related formal asymptotics [1, 20, 26, 38]; some of these treat structures which are suitably asymptotically PE. This relationship is the geometric problem underlying the so called AdS/CFT correspondence of String Theory [51, 57].

The model of a PE manifold is the hyperbolic ball $\mathbb{H}^{d}$ and this yields a large class of further examples via its quotients $\Gamma \backslash \mathbb{H}^{d}$, where $\Gamma$ is a convex cocompact discrete isometry group. There are also various specific examples known in the literature, some of these are catalogued in the work [7] and among these are examples which are not locally conformally flat. Anderson

ARG gratefully acknowledges support from the Royal Society of New Zealand via Marsden Grant no. 06-UOA-029. L was supported by a research fellowship of the Deutsche Forschungsgemeinschaft which enabled him to visit the University of Auckland. FL is grateful to the Department of Mathematics at the UoA for hospitality. 
has rather general existence results in dimension 4 [8]; this extends his earlier works [5, 6]. There are now some rather powerful results available for constructing PE metrics beginning with known ones; in particular through perturbing known examples [39, 12, 46], and via Maskit combinations [52.

Here we describe a product based construction principle for constructing Poincaré-Einstein manifolds. In fact Poincaré-Einstein manifolds are a subclass of the so-called almost Einstein (AE) manifolds as in [30, 32, 33] and the construction applies to this broader setting. Apart from the examples of PE manifolds and Einstein manifolds, almost Einstein structures arise naturally on certain classes of Fefferman spaces [49] (see also [19]) and in the constructions and classifications by Derdzinski and Maschler of Kähler metrics which are "almost everywhere" conformal to Einstein by a non-constant rescaling factor, see e.g. [21, 22] and references therein. The construction we develop enables the proliferation of a variety of classes of examples, and in particular examples of compact PE manifolds and closed (i.e. compact without boundary) AE manifolds. Warped products are well established as a tool for the construction of Einstein metrics [11, 54]. The construction here may be viewed as generalising, or extending, a class of generalised warped products. This extension is made transparent through the fundamental relationship between (almost) Einstein structures and the prolonged differential system captured by the conformal tractor connection. Among the simplest examples, we obtain almost Einstein structures on the sphere products $S^{2} \times S^{1}$ and $S^{3} \times S^{1}$, manifolds well known to not admit Einstein metrics [11].

In 34] for appropriate products of Einstein manifolds we describe the explicit construction of Poincaré-Einstein collar metrics which have the given product as conformal infinity. One may ask which of these may be compactified, that is, which may be embedded into compact PE manifolds. This is essentially answered here in the classification results of Section 4 . We show in Corollary 4.4 that a class of these collars may not be compactified. The remainder, at least given some assumptions concerning simple connectivity, will be seen to arise as conformal infinity neighbourhoods of the examples of Section 3.2, see Theorem 4.5.

All constructions in the following will be on Riemmanian manifolds $(M, g)$ and Riemannian signature conformal manifolds $(M, c)$ (where $c$ is an equivalence class of Riemannian metrics, each pair related by rescaling by a positive function). Many of the statements hold true in other signatures but to simplify the exposition we shall simply avoid this point. Recall that on $\left(M^{d}, g\right)$, with $d \geq 3$, the Schouten tensor $P$ is the trace modification of the Ricci tensor Ric which satisfies Ric $=(d-2) P+g J$, where $J$ is the metric trace of $P$. (Until further notice we shall restrict to manifolds of dimension $d \geq 3$.) We will say that $(M, g, s)$ (or really this modulo an equivalence relation, see Section 2.2) is a directed almost Einstein structure if $s \in C^{\infty}(M)$ is a non-trivial solution to the equation

$$
\text { trace }-\operatorname{free}\left(\nabla^{g} \nabla^{g} s+s P^{g}\right)=0 .
$$

In [32] we see that Poincaré-Einstein manifolds are a special class of directed $\mathrm{AE}$ manifolds. On the other hand, from the following result we see 
that a large class of directed AE manifolds are necessarily boundary gluings of PE manifolds.

Theorem 1.1. 33. Let $\left(M^{d}, g, s\right)$ be a directed almost Einstein structure with $g$ positive definite and $M$ connected. Then writing $\Sigma$ for the zero set of $s, M \backslash \Sigma$ is an open dense set and on this $g^{o}=s^{-2} g$ is Einstein with Ric $=(d-1) S g$. According to the sign of the constant $S=S(g, s)$, there are three cases:

- If $S>0$ then the scale singularity set $\Sigma$ is empty.

- If $S=0$ then $\Sigma$ is either empty or otherwise consists of isolated points and these points are critical points of the function $s$.

- If $S<0$ then $\Sigma$ is either empty or else is a totally umbillic smooth hypersurface. In particular on closed (i.e. $M$ compact without boundary) $S<0$ almost Einstein manifolds, with $\Sigma \neq \emptyset$, a constant rescaling of $s$ normalises $S$ to -1 , and then $\left(M \backslash M^{-}\right)$is a finite union of connected Poincaré-Einstein manifolds. Similar for $\left(M \backslash M^{+}\right)$. Here $M^{ \pm}$is the open submanifold where $s$ is positive, respectively, negative.

Much more can be said about the scale singularity set $\Sigma$ (the conformal infinity of the structure) and its relation to the ambient geometry. This is one of the main directions of 33 . Here the central point is that, from the last item of the above, constructing scalar negative directed AE manifolds is an effective route to PE structures.

If $s$ solves (11) then so does $-s$, and where $s$ is non-vanishing these solutions determine the same Einstein metric. We shall say that a manifold $(M, g)$ is almost Einstein (AE) if it admits a covering such that on each open set $U$ of the cover we have that $\left(U, g, s_{U}\right)$ is directed almost Einstein and on overlaps $U \cap V$ we have either $s_{U}=s_{V}$ or $s_{U}=-s_{V}$. An AE structure will be said to be directable if there is a directed AE structure that agrees locally and up to sign with the given AE structure. Just as the standard conformal sphere yields a model for all categories of directed almost Einstein structure [33] we observe in Section 2.3 that the corresponding conformal structure on $\mathbb{R P}^{d}$ yields a model for $\mathrm{AE}$ structures which are not directable. These yield further examples through the construction principle of Section 3 , see the examples of Section 3.5 .

The tractor connection, due to Thomas [55], is a natural and conformally invariant vector bundle connection equivalent (see [17]) to the normal conformal Cartan connection. As we shall review briefly in the next section, parallel sections of this are in 1-1 correspondence with solutions of (11); in fact more than this, from the construction of the tractor connection in [10] one sees that its equation of parallel transport just is a prolonged system for (11) (cf. [10]). So a directed AE structure is a conformal manifold equipped with a parallel section of the (standard) tractor bundle. More generally an $\mathrm{AE}$ manifold is one which admits a parallel tractor up to a sign in the obvious way. Using this, it follows that a simply connected AE manifold necessarily admits a directed almost Einstein structure.

Each of the closed almost Einstein spaces we construct in Section 3 admits, locally at least, more than one linearly independent parallel tractor; i.e., the conformal class of each admits at least two non-trivially distinct almost Einstein structures. Following some preliminaries, in Sections 2.5 and 2.6 
we investigate the consequences of a fixed conformal structure admitting two linearly independent parallel tractors. This is a natural extension of the problem of conformally related Einstein structures. As in that case, we see here that for closed manifolds the condition of multiple almost Einstein structures is very restrictive. Let us write $\mathcal{K}_{c}$ for the vector space of parallel tractors on a given conformal manifold $(M, c)$. The main result of Section 2.5 is the following.

Theorem 1.2. Let $\left(M^{d}, c\right)$ be a closed Riemannian conformal space of dimension $d \geq 3$ with $\operatorname{dim}\left(\mathcal{K}_{c}\right) \geq 2$. Then either

(1) $(M, c)$ is the standard sphere $\left(S^{d},\left[g_{\mathrm{rd}}\right]\right)$, or

(2) for any $K \in \mathcal{K}_{c} \backslash\{0\}$, it is necessarily the case that $S_{K}<0$ and $\Sigma_{K}$ is non-empty (and hence is a totally umbillic hypersurface in $(M, c))$.

In the Theorem $S_{K}$ is the constant $S$, as in Theorem 1.1, for the almost Einstein structure $\left(M, c, s_{K}\right)$ where $s_{K}$ is determined by the parallel tractor $K$. Note that, in particular, excluding the round sphere, the conformal structure of a closed Einstein manifold never admits a proper directed almost Einstein structure, meaning one with a non-trivial conformal infinity. Equivalently, and again excepting the sphere, on a closed manifold a proper directed almost Einstein structure does not have an Einstein metric in the conformal class. This is part of a picture which indicates that proper almost Einstein structures have in a sense "the same status" as Einstein structures.

It turns out that more can be said about the structure of the scale singularity sets when $\operatorname{dim}\left(\mathcal{K}_{c}\right) \geq 2$.

Theorem 1.3. Let $\left(M^{d}, c\right)$ be a Riemannian conformal space of dimension $d \geq 3$. If $K_{1}, K_{2} \in \mathcal{K}_{c}$ are linearly independent with $S_{K_{2}}<0$ and $\Sigma_{K_{2}} \neq \emptyset$, then on $M \backslash \Sigma_{K_{1}} \Sigma_{K_{2}}$ (is totally umbillic and) has constant mean curvature with respect to the Einstein metric $g^{o}$ determined by $K_{1}$. If the tractors $K_{1}$ and $K_{2}$ are orthogonal then $\Sigma_{K_{2}}$ is minimal (and hence totally geodesic) with respect to the Einstein metric $g^{\circ}$.

We shall prove this in Section 2.6. Related to this we shall see that in fact there is a generalised notion of mean curvature which extends to the conformal infinity $\Sigma_{K_{1}}$ (in the case this is not empty) and this is closely linked to the scalar curvature quantity $S_{K_{1}}$.

Given two Einstein manifolds $\left(M_{1}, g_{1}\right)$ and $\left(M_{2}, g_{2}\right)$, if there is a suitable relationship between the scalar curvatures of each, then we term the product $(M, g)=\left(M_{1} \times M_{2}, g_{1} \times g_{2}\right)$ a special Einstein product. A key observation is that, for such products, the conformal tractor connection is a simple restriction of the direct sum connection. It is convenient for our purposes here to see this directly via the definition and characterisation of the tractor bundle, and this is treated in Proposition 2.13. (See also 50] and 9] where this is observed and is seen to arise naturally in connection with decomposable conformal holonomy. It should be noted that this result also follows easily from the explicit Fefferman-Graham (ambient) metric construction for special Einstein products from Theorem 2.1 of [34] via the Fefferman-Graham metric/tractor relationship established in [18.) Using 
that the tractor connection is essentially a direct sum it follows that a special Einstein product is almost Einstein if and only one of the factors (say $\left.\left(M_{2}, g_{2}\right)\right)$ admits an almost Einstein structure linearly independent of the declared Einstein structure $\left(g_{2}\right)$. Here the "linear independence" refers to the corresponding parallel tractors. This result, Theorem 3.1, is the basis of the construction principle. Also important in this respect is that if one of the factors has a proper almost Einstein structure then the corresponding AE structure on the product is also proper and the singularity set is easily understood, see Corollary 3.2. Away from the singularity set the Einstein metric $g^{o}$ (notation as in Theorem 1.1) is a generalised warped product metric of the form (16). Thus the construction here of almost Einstein manifolds may be viewed as an extension or generalisation of the construction of Einstein manifolds by (suitable) warped products of this form.

The construction via Theorem 3.1 of almost Einstein manifolds that are proper and closed is particularly interesting since then we also obtain compact Poincaré-Einstein spaces via Theorem 1.1. On the other hand the possibilities are limited by Theorem 1.2, for the construction of directed AE special Einstein products one of the factors must be a sphere. Thus the classes of examples developed in Sections 3.2 through to Section 3.4 are exhaustive. Section 3 concludes with some non-directed almost Einstein structures; note that example 3.5 with $m_{1}=1$ shows that any compact Einstein manifold arises as the conformal infinity of a closed almost Einstein manifold. The examples 3.2 give closed conformal manifolds admitting multiple compatible directed AE structures. Generically for these examples the scale singularity sets of the linearly independent parallel tractors intersect. When this happens, if we pick one of these to determine the Einstein metric $g^{o}$ then any other gives a totally umbillic hypersurface (of constant mean curvature in the sense Theorem (1.3) that meets the conformal infinity of $g^{o}$ in a totally umbillic hypersurface (or in isolated double points), see Proposition 2.12. Since this is a way of generating minimal submanifolds that meet conformal infinity it seems that these may be interesting models for branes and the study of their asymptotics (cf. [4, 40]).

\section{Conformal Geometry, tractor calculus and almost Einstein STRUCTURES}

2.1. The conformal tractor bundle. Let $M^{d}$ be a smooth manifold. Until further notice the dimension $d$ is at least 3 . It will be convenient to use some standard structures from conformal geometry, further details and background may be found in [18, 35. Recall that a (Riemannian) conformal structure on $M$ is a smooth ray subbundle $\mathcal{Q} \subset S^{2} T^{*} M$ whose fibre over $p \in M$ consists of conformally related positive definite metrics at the point $p$. Sections of $\mathcal{Q}$ are metrics $g$ on $M$. So we may equivalently view the conformal structure as the equivalence class $c=[g]$ of these conformally related metrics. The principal bundle $\pi: \mathcal{Q} \rightarrow M$ has structure group $\mathbb{R}_{+}$, and so each representation $\mathbb{R}_{+} \ni t \mapsto t^{-w / 2} \in \operatorname{End}(\mathbb{R})$ induces a natural line bundle on $(M, c)$ that we term the conformal density bundle $\mathcal{E}[w]$. We write $\mathcal{E}^{a}$ as an abstract index notation for the tangent bundle $T M$ and similarly $\mathcal{E}_{a}$ is an alternative notation for the cotangent bundle $T^{*} M$. In general each 
vector bundle and its space of smooth sections will be denoted by the same notation.

We write $\boldsymbol{g}$ for the conformal metric, that is the tautological section of $S^{2} T^{*} M[2]:=S^{2} T^{*} M \otimes \mathcal{E}[2]$ determined by the conformal structure. This will be henceforth used to identify $T M$ with $T^{*} M[2]$. For example, with these conventions the Laplacian $\Delta$ is given by $\Delta=-\boldsymbol{g}^{a b} \nabla_{a} \nabla_{b}=-\nabla^{b} \nabla_{b}$ where $\nabla$ (or sometimes we will write $\nabla^{g}$ ) is the Levi-Civita connection for some choice of metric $g$ from the conformal class. Note $\mathcal{E}[w]$ is trivialised by a choice of metric, and we write $\nabla$ (or again sometimes $\nabla^{g}$ ) for the connection corresponding to this trivialisation. It follows immediately that the (coupled) connection $\nabla_{a}$ preserves the conformal metric and so the canonical conformal volume form (of conformal weight $n$ ) is also parallel for $\nabla_{a}$. The conformal metric $\boldsymbol{g}$ and its inverse will henceforth be the default object used to contract indices on tensors even when we have fixed a metric from the conformal class.

The Riemann curvature tensor $R_{a b}{ }^{c}{ }_{d}$ is given by

$$
\left(\nabla_{a} \nabla_{b}-\nabla_{b} \nabla_{a}\right) V^{c}=R_{a b}^{c}{ }^{c} V^{d} \quad \text { where } \quad V^{c} \in \mathcal{E}^{c} .
$$

This can be decomposed into the totally trace-free Weyl curvature $C_{a b c d}$ and the symmetric Schouten tensor $P_{a b}$ according to

$$
R_{a b c d}=C_{a b c d}+2 g_{c[a} P_{b] d}+2 g_{d[b} P_{a] c} .
$$

Changing to a conformally related metric $\widehat{g}=e^{2 \omega} g$ ( $\omega$ a smooth function) one finds the Weyl tensor is conformally invariant $\widehat{C}_{a b}^{c} d=C_{a b}{ }^{c} d$, whereas the Schouten tensor transforms to

$$
\widehat{\mathrm{P}}_{a b}=\mathrm{P}_{a b}-\nabla_{a} \Upsilon_{b}+\Upsilon_{a} \Upsilon_{b}-\frac{1}{2} \Upsilon^{c} \Upsilon_{c} \boldsymbol{g}_{a b}
$$

where $\Upsilon:=d \omega$.

For a given choice of metric $g$, the (standard) tractor bundle $\mathcal{T}$, or using an abstract index notation $\mathcal{E}^{A}$, may be identified with a direct sum

$$
\mathcal{T}=\mathcal{E}^{A} \stackrel{g}{=} \mathcal{E}[1] \oplus \mathcal{E}_{a}[1] \oplus \mathcal{E}[-1] .
$$

Thus a section $U$ of $\mathcal{T}$ may be identified with a triple $\left(\sigma, \mu_{a}, \rho\right)$; when it is understood that a metric $g$ has been fixed we will write simply $U^{A}=$ $\left(\sigma, \mu_{a}, \rho\right)$. Conformally transforming, as above, gives a different isomorphism, which is related to the previous by the transformation formula

$$
\left(\widehat{\sigma, \mu_{b}, \rho}\right)=\left(\sigma, \mu_{b}+\sigma \Upsilon_{b}, \rho-\boldsymbol{g}^{b c} \Upsilon_{b} \mu_{c}-\frac{1}{2} \sigma \boldsymbol{g}^{b c} \Upsilon_{b} \Upsilon_{c}\right) .
$$

It follows that $\mathcal{T}$ invariantly has a composition structure

$$
\mathcal{T}=\mathcal{E}[1] \oplus \mathcal{E}_{a}[1] \oplus \mathcal{E}[-1] ;
$$

$\mathcal{E}[-1]$ may be identified with a subbundle of $\mathcal{T}$ and $\mathcal{E}_{a}[1]$ is a subbundle of the quotient bundle $\mathcal{T} / \mathcal{E}[-1]$. From (3) one also sees that the map $\mathcal{E}^{A} \rightarrow \mathcal{E}[1]$ is conformally invariant and may be regarded as a preferred section $X_{A}$ of $\mathcal{E}_{A}$ [1] so that, with $U^{A}$ as above, we have $\sigma=U^{A} X_{A}$. This section also describes the invariant injection $\mathcal{E}[-1] \rightarrow \mathcal{E}_{A}$ according to $\rho \mapsto \rho X_{A}$. In computations, it is often useful to introduce the remaining 'projectors' $Z_{A a}: \mathcal{E}^{A} \rightarrow \mathcal{E}_{a}[1]$ and $Y_{A}: \mathcal{E}^{A} \rightarrow \mathcal{E}[-1]$. which are determined by a choice of scale. For calculations it is useful to view these as sections $Z_{A a} \in \mathcal{E}_{A a}[1]$ and $Y_{A} \in \mathcal{E}_{A}[-1]$, where $\mathcal{E}_{A a}[w]=\mathcal{E}_{A} \otimes \mathcal{E}_{a} \otimes \mathcal{E}[w]$, and so forth. 
We describe any tensor product (or symmetric tensor product etcetera) of the tractor bundle and its dual as tractor bundles. If such a bundle is tensored with some bundle of densities $\mathcal{E}[w]$ then we shall describe the result as a weighted tractor bundle. Repeated (abstract) tractor indices indicate a contraction, just as for tensor indices.

The bundle $\mathcal{E}^{A}$ carries an invariant signature $(d+1,1)$ tractor metric $h_{A B}$, and a connection $\nabla_{a}$ which preserves this. For $U^{A}$ as above, this metric is determined by

$$
h_{A B} U^{A} U^{B}=2 \rho \sigma+\mu^{a} \mu_{a} .
$$

As a point on notation, we may also write $h(U, U)$ for the expression in the display. The tractor metric will be used to raise and lower indices without further mention. In terms of the metric $g$ from the conformal class, the tractor connection is given explicitly by the following formula for $\nabla_{a} U^{B}$ :

$$
\nabla_{a}\left(\begin{array}{c}
\sigma \\
\mu^{b} \\
\rho
\end{array}\right)=\left(\begin{array}{c}
\nabla_{a} \sigma-\mu_{a} \\
\nabla_{a} \mu^{b}+\delta_{a}^{b} \rho+P_{a}^{b} \sigma \\
\nabla_{a} \rho-P_{a c} \mu^{c}
\end{array}\right) .
$$

This gives the normal tractor connection, in all statements below the tractor connection may be assumed to be this one, unless otherwise stated. Of course this may be extended to a connection on any tractor bundle in the obvious way. The use of the same symbol $\nabla$ as for the Levi-Civita connection is intentional. More generally, we shall use $\nabla$ to mean the coupled Levi-Civita-tractor connection: this enables us, for example, to apply $\nabla$ to weighted tractor bundles or tensor-tractor. Although in this case it is not conformally invariant it enables us to, for example, compute the covariant derivative of the tractor projectors $X, Y$ and $Z$.

As discussed in [10], there is an invariant second order operator between weighted tractor bundles due to T.Y. Thomas,

$$
D_{A}: \mathcal{T}^{*}[w] \rightarrow \mathcal{T}^{*}[w-1], \quad \mathcal{T}^{*} \text { any tractor bundle, }
$$

given by

(6) $D_{A} V:=(d+2 w-2) w Y_{A} V+(d+2 w-2) Z^{a} \nabla_{a} V+X^{A}(\Delta-w J) V$.

For an invariant construction of this see [29, 16].

2.2. Almost Einstein structures. As above we work on Riemannian manifolds $\left(M^{d}, g\right)$ with the dimension $d \geq 3$. The Schouten tensor $P\left(\right.$ or $P^{g}$ ), introduced earlier, is related to the Ricci tensor by Ric $=(d-2) P+J g$, where recall $J$ is the conformal metric trace of $P^{g}$. Calculating the conformal transformation of $P$, it follows easily that the metric $g$ is conformally Einstein if and only if there is a nowhere zero solution $s \in C^{\infty}(M)$ to the equation (11) trace $-\operatorname{free}(\nabla \nabla s+P s)=0$.

Note that the equation (11) is conformally covariant. If we write $A(g, s):=$ trace $-\operatorname{free}(\nabla \nabla s+P s)$ then $e^{\omega} A(g, s)=A\left(e^{2 \omega} g, e^{\omega} s\right), \omega \in C^{\infty}(M)$. By a directed almost Einstein structure we mean $(M, g, s)$, where $s$ is a solution of (1), but modulo the equivalence relation $(g, s) \sim\left(e^{2 \omega} g, e^{\omega} s\right)$; more precisely it is an equivalence class $[(M, g, s)]$ where the equivalence relation is $(M, g, s) \sim\left(M, e^{2 \omega} g, e^{\omega} s\right)$ and $s$ is a solution of (1). 
The conformal covariance of the equation (1) is equivalent to conformal invariance, if we replace the function $s$ by a conformal density $\sigma \in \mathcal{E}[1]$. The equation

$$
\text { trace }-\operatorname{free}\left(\nabla_{a} \nabla_{b} \sigma+P_{a b} \sigma\right)=0
$$

is conformally invariant. This suggests a strong link to conformal geometry. As observed in [30] it follows easily from the definition (5) of the tractor connection above that we have the following.

Theorem 2.1. A directed almost Einstein structure is a conformal manifold $(M, c)$ equipped with a parallel (standard) tractor $I \neq 0$. The mapping from non-trivial solutions of (7) to parallel tractors is by $\sigma \mapsto \frac{1}{d} D \sigma$ with inverse $I \mapsto \sigma:=h(I, X)$, and $\sigma$ is non-vanishing on an open dense set $M \backslash \Sigma$. On this set $g^{o}:=\sigma^{-2} \boldsymbol{g}$ is Einstein.

The point here, as touched on earlier, is the parallel transport equation for the tractor connection (15) is simply a prolonged system derived from (and essentially equivalent to) (7) [10, 33. So a directed almost Einstein manifold is exactly a conformal manifold $(M, c)$ equipped with a parallel standard tractor $I$ and we write $(M, c, I)$ to indicate this. The set $\Sigma$, where the almost Einstein "scale" $\sigma:=h(X, I)$ vanishes, shall be called the scale singularity set.

On an almost Einstein manifold $(M, c, I)$ (or $(M, g, s)$ ) we shall write $S_{I}$ or $S(\sigma)$ (or even $S(g, s)$ ) as a shorthand for $-h(I, I)$. This may be viewed as a scalar curvature quantity for the structure, since off $\Sigma$ we have $S(\sigma)=\frac{\mathrm{Sc}^{g^{o}}}{d(d-1)}$, and this is the quantity $S$ in Theorem 1.1. In this language that theorem is as follows.

Theorem 2.2. 33 Let $(M, c, I)$ be a Riemannian signature almost Einstein structure and $\sigma=h(X, I)$. If $S(\sigma)>0$ then $\Sigma$ is empty and $\left(M, \sigma^{-2} \boldsymbol{g}\right)$ is Einstein with positive scalar curvature; If $S(\sigma)=0$ then $\Sigma$ is either empty or consists of isolated points $p$ where $j_{p}^{1} \sigma=0$, and $\left(M \backslash \Sigma, \sigma^{-2} \boldsymbol{g}\right)$ is Ricciflat; if $S(\sigma)<0$ then the scale singularity set $\Sigma$ is either empty or else is a totally umbillic smooth hypersurface, and $\left(M \backslash \Sigma, \sigma^{-2} \boldsymbol{g}\right)$ is Einstein of negative scalar curvature.

The Theorem here is a straightforward consequence of the formulae (5) and (41). There is a detailed treatment of this and surrounding results in [33. Note that if $\Sigma \neq \emptyset$ then $\Sigma$ is a conformal infinity for the ambient Einstein metric $g^{o}$ in the usual sense; there is a metric $g$ on the manifold $M$ so that $g^{o}=s^{-2} g$. In this situation it is well known that if $M$ is complete then $\left(M \backslash \Sigma, g^{o}\right)$ is geodesically complete. In the case that $M$ is compact it is traditional to describe $\left(M \backslash \Sigma, g^{o}\right)$ as conformally compact. If also $S(\sigma)<0$ then the smooth hypersurface $\Sigma$ inherits the conformal structure $\left(\Sigma,\left[\left.g\right|_{\Sigma}\right]\right)$.

In terms of the tractor picture to say that we have an almost Einstein manifold that is not necessarily directed means that on each open set $U$ of some cover we have $\left(M, c, I_{U}\right)$, with $I_{U}$ parallel, and on overlaps $U \cap V$ we have $I_{U}= \pm I_{V}$. We will write $(M, c,[I])$, or simply $[I]$ if the conformal structure is fixed, to denote such an $\mathrm{AE}$ structure. 
2.3. The Models. On the $d$-sphere, with its standard conformal structure, the standard tractor bundle may be trivialised globally by parallel sections. Thus we have the following.

Proposition 2.3. The d-sphere, with its standard conformal structure, admits a $(d+2)$-dimensional space of compatible directed almost Einstein structures.

In fact for each $S \in \mathbb{R}$ there is an almost Einstein structure $I$ on $\mathbb{S}^{d}$ with $S_{I}=S$, this is Proposition 5.1 in [33] and the case of the sphere as a model for directed almost Einstein structures is treated in detail there.

The standard conformal structure on the sphere is recovered as follows. Consider a $(d+2)$-dimensional real vector space $\mathbb{V}$ equipped with a nondegenerate bilinear form $\mathcal{H}$ of signature $(d+1,1)$. The null cone $\mathcal{N}$ of zerolength vectors form a quadratic variety. Let us write $\mathcal{N}_{+}$for the forward part of $\mathcal{N} \backslash\{0\}$. Under the ray projectivisation of $\mathbb{V}$ the forward cone $\mathcal{N}_{+}$is mapped to a quadric in $\mathbb{P}_{+}(\mathbb{V}) \cong \mathbb{S}^{d+1}$. This image is topologically a sphere $\mathbb{S}^{d}$ and we will write $\pi$ for the submersion $\mathcal{N}_{+} \rightarrow \mathbb{S}^{d}$. Each point $p \in \mathcal{N}_{+}$determines a positive definite inner product on $T_{x=\pi p} \mathbb{S}^{d}$ by $g_{x}(u, v)=$ $\mathcal{H}_{p}\left(u^{\prime}, v^{\prime}\right)$ where $u^{\prime}, v^{\prime} \in T_{p} \mathcal{N}_{+}$are lifts of $u, v \in T_{x} \mathbb{S}^{d}$. For a given vector $u \in T_{x} \mathbb{S}^{d}$ two lifts to $p \in \mathcal{N}_{+}$differ by a vertical vector field. Since any vertical vector is orthogonal (with respect to $\mathcal{H}$ ) to the cone it follows that $g_{x}$ is independent of the choices of lifts. Clearly then, each section of $\pi$ determines a metric on $\mathbb{S}$ and by construction this is smooth if the section is. (Evidently the metric agrees with the pull-back of $\mathcal{H}$ via the section concerned.) Now, viewed as a metric on $T \mathbb{R}^{d+2}, \mathcal{H}$ is homogeneous of degree 2 with respect to the standard Euler vector field $E$ on $\mathbb{V}$. That is $\mathcal{L}_{E} \mathcal{H}=2 \mathcal{H}$, where $\mathcal{L}$ denotes the Lie derivative. In particular, this holds on the cone, and we note also that the cone is generated by $E$.

As explained in [33. (and cf. [18]), the tractor bundle on $\mathbb{S}^{d}$ is then naturally identified with $\left.T \mathbb{V}\right|_{\mathcal{N}_{+}} / \sim$. Here the equivalence relation " $\sim$ " is as follows: for $p, q \in \mathcal{N}_{+}$we have $T_{p} \sim T_{q}$ if and only if $p$ and $q$ lie in the same null ray through the origin and $T_{p}$ is parallel to $T_{q}$ according to the usual parallelism of $\mathbb{V}$ as an affine space. The tractor connection is induced from this same parallelism, and the usual tractor metric $h$ is induced from $\mathcal{H}$.

It follows that each vector $I$ in $\mathbb{V}$ determines a parallel tractor field on $\mathbb{S}^{d}$. In particular if we fix a choice $I$ with $|I|^{2}=-1$ the we obtain a standard round metric on the sphere; we shall write $\left(S^{d}, g\right)$ for this. This can be viewed as arising from the section of $\mathcal{N}_{+}$given by the intersection with the hyperplane $I_{A} X^{A}=1$, where $X^{A}$ are standard coordinates on $\mathbb{V} \cong \mathbb{R}^{d+2}$. Let us write $I^{\perp}$ for the vectors in $\mathbb{V}$ orthogonal to $I$ according to $\mathcal{H}$ and then also use the same notation for the corresponding tractors. It is easily verified that the tractors $K \in I^{\perp}$ correspond to solutions of (7) that are odd with respect to $\left(S^{d}, g\right) ; \alpha:=h(K, X)$ takes the opposite sign at antipodal points. Let us now form from $\left(S^{d}, g\right)$ the unit $\mathbb{R P}^{d}$ which we shall denote by $\left(\mathbb{R P}^{d}, g\right)$ by taking an antipodal identification of $\left(S^{d}, g\right)$. It is clear that each $K \in I^{\perp}$ descends to an almost Einstein structure $[K]$ on $\left(\mathbb{R} \mathbb{P}^{n}, g\right)$ that is not directed. Thus we have the following. 
Proposition 2.4. $\mathbb{R P}^{d}$ with its standard conformal structure, admits an Einstein metric and also a $(d+1)$-dimensional space of compatible almost Einstein structures that are not directed.

2.4. Conformal Killing vectors and tractors. Via Theorem 2.1 the equation of tractor parallel transport captures the basic equation (11) in a geometric form that plays a central role in our discussions below. The prolonged system corresponding to conformal Killing vectors is also handled well by the tractor machinery. First note that (as observed in [23]) the operator $M_{A}{ }^{a}: \mathcal{E}_{a}[w] \rightarrow \mathcal{E}_{A}[w-1]$ given, in a conformal scale by

$$
u_{a} \mapsto(d+w-2) Z_{A}^{a} u_{a}-X_{A} \nabla^{a} u_{a},
$$

is conformally invariant. Then by Lemma 2.1 in 31] we have that solutions $k^{a}$ of the conformal Killing equation

$$
\nabla_{(a} k_{b)_{0}}=0
$$

are in 1-1 correspondence with fields $K_{B} \in \mathcal{E}_{B}[1]$ such that

$$
D_{A} K_{B}=\mathbb{K}_{A B}
$$

where $\mathbb{K}_{A B}$ is skew (i.e. it is a 2-form tractor). The correspondence is given by

$$
k^{a} \mapsto \frac{1}{d} M_{B a} k^{a} \quad \text { with inverse } \quad K_{B} \mapsto Z^{B a} K_{B} .
$$

Using the above (or otherwise [15, 36]), the conformal Killing condition is also captured by an equation on the skew part $\mathbb{K}_{A B}$ of $D_{A} K_{B}$ as follows.

Proposition 2.5. [31, Proposition 3.1] Solutions $k^{a}$ of the conformal Killing equation

$$
\nabla_{(a} k_{b)_{0}}=0
$$

are in 1-1 correspondence with solutions $\mathbb{K}_{D E} \in \mathcal{E}_{[D E]}$ of the equation

$$
\nabla_{a} \mathbb{K}_{D E}-\mathbb{K}_{A B} X^{A} Z^{B a} \Omega_{a b D E}=0 .
$$

The correspondence is given by

$$
k^{a} \mapsto \frac{1}{d^{2}} D_{[A} M_{B] a} k^{a} \quad \text { with inverse } \quad \mathbb{K}_{A B} \mapsto X^{A} Z^{B a} \mathbb{K}_{A B} .
$$

If $\mathbb{K}_{A B}$ is a parallel adjoint tractor then $k^{a}:=X^{A} Z^{B a} \mathbb{K}_{A B}$ is a conformal Killing vector field and hence, from (9), satisfies $k^{a} \Omega_{a b D E}=0$.

Given $k_{a} \in \mathcal{E}[2]$ the 2 -form tractor $\mathbb{K}_{A B}$ is given by

$$
\mathbb{K}_{A B} \underline{g}=\left(\begin{array}{cc}
k_{a} & \frac{1}{d} \nabla^{c} k_{c} \\
\nabla_{[a} k_{b]} & \frac{1}{d}\left(\nabla^{c} \nabla_{(c} k_{a)_{0}}-\nabla_{a} \nabla^{c} k_{c}\right)-P_{a}^{c} k_{c}
\end{array}\right)
$$

where from the top-left to right-bottom the entries are the coefficients of $\mathbb{Y}_{A B}=Y_{[A} Z_{B]}^{a}, \mathbb{Z}_{A B}^{a b}=Z_{[A}^{a} Z_{B]}^{b}, \mathbb{W}_{A B}=X_{[A} Y_{B]}, \mathbb{X}_{A B}=X_{[A} Z_{B]}^{a}$.

We say a conformal vector field on a space $(M, c)$ is essential if it is not a Killing vector with respect to any metric $\tilde{g} \in c$ on $M$, i.e.

$$
L_{V} \tilde{g}=\frac{2}{d} \operatorname{div}_{\tilde{g}}(V) \tilde{g} \neq 0 \quad \text { for any } \tilde{g} \in c .
$$


Note that every essential conformal vector field $V$ necessarily has a zero at some point; a conformal Killing vector $V$ without a zero is Killing for the conformally changed metric $\frac{1}{g(V, V)} g$.

We call a conformal Killing vector field $k$ on $(M, g)$ a conformal gradient if there exists a smooth function $\psi$ on $M$ such that $k=\operatorname{grad}_{g}(\psi)$. Note that this is not a conformally invariant property; $k$ is not a gradient for other metrics in the conformal class.

Theorem 2.6. On a Riemannian manifold, of dimension at least 3, a conformal gradient with at least one zero is an essential conformal vector field.

Proof: If $k$ is a gradient then in any scale we have $\nabla_{[a} k_{b]}=u_{[a} k_{b]}$ for some 1-form $u$. If we assume also that $k$ is not essential and for $p \in M$ we have $k(p)=0$ then it follows that the tractor 2 -form $\mathbb{K}_{A B}$ (of (10)) is zero at $p$. But the left hand side of (9) determines a connection (which is a modification of the normal tractor connection) for which this tractor $\mathbb{K}$ is parallel. So $\mathbb{K}$ is zero everywhere and hence so is $k$.

In fact this result is well known and also holds in dimension 2 44] (although we shall use it only in higher dimensions); we include it here for completeness since, as seen, it follows easily from Proposition 2.5.

2.5. Multiple almost Einstein structures. Here we want to consider the situation where a given fixed conformal structure admits linearly independent parallel standard tractors, or equivalently, linearly independent solutions of (7). Note that in general these are not "conformally related", as two solutions will in general have different zero sets. Of course if $\sigma_{1}$ and $\sigma_{2}$ are two such solutions, then away from the union of the two zero sets $\sigma_{1}^{-2} \boldsymbol{g}$ and $\sigma_{2}^{-2} \boldsymbol{g}$ are conformally related Einstein metrics.

It is well known that a pair of conformally related Einstein metrics determines a conformal gradient field [13]. We observe here that this generalises (adapting and extending an argument from Theorem 2.4 of [31]).

Theorem 2.7. If $g_{1}=\left(\sigma_{1}\right)^{-2} \boldsymbol{g}$ is an Einstein metric, then $\left(M,\left[g_{1}\right], \sigma_{2}\right)$ is an almost Einstein structure if and only if the vector field

$$
k^{a}:=\sigma_{1} \nabla^{a} \sigma_{2}-\sigma_{2} \nabla^{a} \sigma_{1}
$$

is a conformal gradient field with respect to $g_{1}$.

Proof: $\Rightarrow$ : Since $\sigma_{1}$ and $\sigma_{2}$ are almost Einstein scales it follows from Theorem 2.1 that

$$
I_{1}^{A}:=\frac{1}{n} D^{A} \sigma_{1} \quad \text { and } I_{2}^{A}:=\frac{1}{n} D^{A} \sigma_{2}
$$

are both parallel for the tractor connection. Thus

$$
\mathbb{K}^{A B}:=I_{1}^{A} I_{2}^{B}-I_{1}^{B} I_{2}^{A} \stackrel{\underline{g}}{=}\left(\begin{array}{c}
\sigma_{1} \nabla^{a} \sigma_{2}-\sigma_{2} \nabla^{a} \sigma_{1} \\
\left(\nabla_{[a} \sigma_{1}\right) \nabla_{b]} \sigma_{2} \\
\frac{1}{d}\left(\sigma_{2} \Delta \sigma_{1}-\sigma_{1} \Delta \sigma_{2}\right) \\
\frac{1}{d}\left(\left(\Delta \sigma_{1}\right) \nabla_{a} \sigma_{2}-\left(\Delta \sigma_{2}\right) \nabla_{a} \sigma_{1}+J\left(\sigma_{2} \nabla_{a} \sigma_{1}-\sigma_{1} \nabla_{a} \sigma_{2}\right)\right)
\end{array}\right)
$$

is a parallel tractor 2-form. On the right $g$ is any metric from the conformal class $\left[g_{1}\right]=\left[g_{2}\right]$ and $\nabla_{b}$ is the corresponding Levi-Civita connection. By 
Proposition 2.5 the primary part

$$
k^{b}:=X_{A} Z_{B}^{b} \mathbb{K}^{A B}=\sigma_{1} \nabla^{b} \sigma_{2}-\sigma_{2} \nabla^{b} \sigma_{1}
$$

is a conformal Killing field. On the other hand since $\sigma_{1}$ is nowhere vanishing we may work in the scale $g_{1}=\sigma_{1}^{-2} \boldsymbol{g}$; with $\nabla$ the corresponding Levi-Civita connection, we have $\nabla \sigma_{1}=0$. Thus we see that $k^{b}$ is a gradient:

$$
k^{b}=g_{1}^{b c} \nabla_{c} s
$$

where $s$ is the function $\sigma_{2} / \sigma_{1}$. Note that away from the zero set of $s$, $g_{2}=s^{-2} g_{1}$ is Einstein.

$\Leftarrow$ : By definition $g_{2}$ is almost Einstein if and only if $\nabla_{(a}^{g_{1}} \nabla_{b)_{0}}^{g_{1}} \sigma_{2}+P_{(a b)_{0}}^{g_{1}} \sigma_{2}=$ 0 (see (10)). Now recall that $\nabla_{a}^{g_{1}} \sigma_{1}=0$. Using this and that $\sigma_{1}$ is nonvanishing, we see that $\nabla_{(a} k_{b)_{0}}=0$ implies $\nabla_{(a}^{g_{1}} \nabla_{b)_{0}}^{g_{1}} \sigma_{2}=0$. On the other hand since $g_{1}$ is Einstein $P_{(a b)_{0}}^{g_{1}}=0$.

Remark: Note that not every conformal gradient comes from a $\nabla$-parallel 2 -form tractor. III

By an obvious variation of the proof above we have the following.

Proposition 2.8. If $\left(M, c, \sigma_{1}\right)$ is an almost Einstein structure then $\left(M, c, \sigma_{2}\right)$ is also an almost Einstein structure if and only if the vector field

$$
k^{a}:=\sigma_{1} \nabla^{a} \sigma_{2}-\sigma_{2} \nabla^{a} \sigma_{1}
$$

is a conformal Killing vector field.

Remark: Note that $k^{a}$ in (13) is not a gradient if there is any point where both $\sigma_{1}$ and $\sigma_{2}$ vanish. One might term such a vector $k^{a}$ an almost conformal gradient.

2.6. Multiple almost Einstein structures: geometric implications. We will see from the explicit formula (12) that the existence of two linearly independent almost Einstein structures in a given fixed conformal class has strong implications for the geometry and topology of the underlying space. This uses some powerful results concerning essential conformal vector fields.

Theorem 2.9. 2, 27, 58] Let $\left(M^{d}, g\right), d \geq 2$, be a Riemannian space, without boundary, admitting a complete and essential conformal vector field.

(1) If $(M, g)$ is non-compact then it is conformal to the Euclidean space $\mathbb{R}^{d}$.

(2) If $(M, g)$ is compact then it is conformal to the sphere $S^{d}$ with round metric $g_{r d}$.

The case $d=2$ of the above is classically known from the theory of Riemann surfaces. Theorem 2.9] says, in particular, that any Riemannian space with a complete essential vector field is conformally flat.

Theorem 2.10. Let $\left(M^{d}, g\right), d \geq 2$, be a closed Riemannian manifold which admits a conformal gradient. Then $\left(M^{d}, g\right)$ is conformal to the round sphere $\left(S^{d}, g_{r d}\right)$.

Proof: Any gradient on $M$ has zeros, since any function on a compact manifold has critical points. However by Theorem 2.6 a conformal gradient field with a zero is essential. So the result follows from Theorem 2.9. 
Proposition 2.11. Suppose that $\left(M^{d \geq 3}, c\right)$ admits two linearly independent parallel standard tractors $I_{1}$ and $I_{2}$, where $I_{1}$ is scalar flat and $\sigma_{1}=h\left(X, I_{1}\right)$ has a zero at $p \in M$. Then the primary part $k:=\sigma_{1} \nabla^{a} \sigma_{2}-\sigma_{2} \nabla^{a} \sigma_{1}$ of the parallel $\frac{1}{2} I_{1} \wedge I_{2}$ is an essential conformal Killing field.

Proof: Note that since $I_{1}$ and $I_{2}$ are linearly independent we have $I_{1} \wedge I_{2} \neq$ 0 . Since by Theorem 2.2 (or equivalently Theorem 1.1) $j_{p}^{1} \sigma_{1}=0$ it follows from (12) that the 2-form tractor $\frac{1}{2} I_{1} \wedge I_{2}$ is given at $p \in M$ by

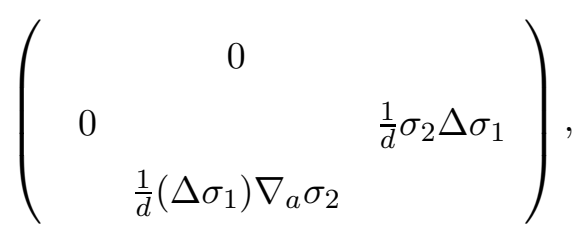

with respect to any metric $\tilde{g} \in c$. This shows that $k$ has a zero at $p$, and (by (10)) either the divergence $\nabla_{c} k^{c}$ or $\nabla_{a} \nabla_{c} k^{c}$ does not vanish at $p$. Since $\tilde{g}$ was an arbitrary metric from the conformal class it follows that the primary part $k$ of $\frac{1}{2} I_{1} \wedge I_{2}$ is an essential conformal vector field.

Next note that if $I_{1}$ and $I_{2}$ are linearly independent scalar negative almost Einstein structures such that zero sets of the scales $\sigma_{1}$ and $\sigma_{2}$ intersect nontrivially then the projecting part $k=\sigma_{1} \nabla^{a} \sigma_{2}-\sigma_{2} \nabla^{a} \sigma_{1}$ of $\frac{1}{2} I_{1} \wedge I_{2}$ has a zero on $(M, c)$. However, the vector $k$ is only an almost gradient field, in the sense of the Remark in Section 2.5, and so we cannot argue that $k$ is essential.

Using our observations so far we led to the characterisation of compact Riemannian spaces with at least 2 linearly independent directed almost Einstein structures as given in Theorem 1.2 .

Proof of Theorem 1.2. Let $I_{1}$ and $I_{2}$ be two linearly independent parallel tractors on $(M, c)$ and assume that $I_{1}$ is Einstein that is $\sigma_{1}:=h\left(X, I_{1}\right)$ is nowhere vanishing. Then, from Theorem 2.7, the projecting part $k$ of $\frac{1}{2} I_{1} \wedge I_{2}$ is a conformal gradient with respect to $g_{1}:=\sigma_{1}^{-2} \boldsymbol{g}$ and so the result follows from Theorem 2.10.

Now let us assume that $(M, c)$ is not conformally Einstein but that there exists a proper almost Ricci-flat structure, that is a null tractor $I_{1}$ with $h\left(X, I_{1}\right)$ zero at some point. For any linearly independent parallel standard tractor $I_{2}$ (from the class assumed to exist on $(M, c)$ ) it follows from Proposition 2.11 that the primary part of $\frac{1}{2} I_{1} \wedge I_{2}$ is essential. From Theorem 2.9 we can again conclude that $(M, c)$ is conformal to the round sphere.

The remaining possibility is that every parallel standard tractor $K$ on $(M, c)$ is scalar negative and has a non-empty scale singularity set $\Sigma_{K}$. But then from Theorem 2.2 (or equivalently Theorem 1.1) it follows that $\Sigma_{K}$ is a totally umbillic hypersurface.

Note that a result by S. Gallot states that if the Riemannian cone of a compact Riemannian space $(M, g)$ has decomposable holonomy then $(M, g)$ is isometric to the round sphere [28]. Observe that this is consistent with the statement of Theorem 1.2 as follows. In the case of an Einstein space with positive scalar curvature the Fefferman-Graham ambient metric is just a product of the cone with a line [37, 48, and it follows (see [34]) that almost Einstein structures are just parallel vectors on the cone. On the other hand parallel vectors on the cone mean that the holonomy is decomposable. 
Before beginning the construction of examples we pause to prove the other result from the introduction.

Proof of Theorem 1.3. Since $K_{1}$ and $K_{2}$ are parallel, and the tractor connection preserves the tractor metric, it follows that $h\left(K_{1}, K_{2}\right)$ is constant on $M$ (at least if $M$ is connected, as we assume for simplicity). On $M \backslash \Sigma_{K_{1}}$ $\sigma_{1}:=h\left(X, K_{1}\right)$ is non-vanishing and we may work in the metric scale $g^{o}=$ $\sigma_{1}^{-2} \boldsymbol{g}$. There and then we have

$$
K_{1} \stackrel{g^{o}}{=}\left(\sigma_{1}, 0,-\frac{1}{d} \mathrm{~J}^{g^{o}} \sigma_{1}\right) .
$$

On the other hand from [33, Proposition 3.6] along $\Sigma_{K_{2}}, K_{2}$ agrees with the normal tractor (of [10, Definition 2.8])

$$
\left.K_{2}\right|_{\Sigma_{K_{2}}}=N_{\Sigma_{K_{2}}} \stackrel{g^{o}}{=}\left(0, \nabla^{g^{o}} \sigma_{2},-H^{g^{o}}\right),
$$

where $\sigma_{2}:=h\left(X, K_{2}\right)$ and $H^{g^{o}}$ is the mean curvature of $\Sigma_{K_{2}}$ in the metric scale $g^{o}$. Now in fact $H^{g^{o}}$ here is the mean curvature as a weight -1 conformal density. The mean curvature as a function in the scale $g^{o}$ is $\underline{H}^{g^{o}}=\sigma_{1} H^{g^{o}}$. But from the last two displays this is exactly (the negative of) the tractor inner product of $K_{1}$ with $N_{\Sigma_{K_{2}}}$. That is:

$$
\underline{H}^{g^{o}}=-h\left(K_{1}, N_{\Sigma_{K_{2}}}\right) .
$$

But, as remarked above, the conformally invariant inner product $h\left(K_{1}, K_{2}\right)$ is constant on $M$, while evidently along $\Sigma_{K_{2}}$ we have $h\left(K_{1}, K_{2}\right)=h\left(K_{1}, N_{\Sigma_{K_{2}}}\right)$. So we may conclude that

$$
\underline{H}^{g^{o}}=-h\left(K_{1}, K_{2}\right),
$$

and this is constant. Note that in particular, this constant is zero if $K_{1}$ and $K_{2}$ are orthogonal.

Remark: Note that it is clear, from our considerations in the proof immediately above, that given an almost Einstein structure $(M, c, K)$ and a smooth hypersurface $\Sigma$ with normal tractor $N$, then $-h(K, N)$ gives a function along $\Sigma$ which generalises the mean curvature: it is defined on all of $\Sigma$ and is exactly the mean curvature $H^{g^{\circ}}$ on $M \backslash \Sigma_{K}$. Here $\Sigma_{K}$ is the zero set of $\sigma_{K}:=h(K, X)$ and $g^{o}=\sigma_{K}^{-2} \boldsymbol{g}$. In fact this idea generalises beyond almost Einstein spaces, but this will be taken up elsewhere.

Next observe that if we have $K_{1}$ and $K_{2}$ as in the Theorem 1.3 and we write $K_{t}:=\cos t K_{1}+\sin t K_{2}$ then (away from the zero set of $\sigma_{1}=h\left(X, K_{1}\right)$ ) for $\mathbb{R} \ni t \notin \pi \mathbb{Z}$ we have

$$
h\left(K_{1}, K_{t}\right)=-\underline{H}_{\Sigma_{K_{t}}}^{g^{o}}
$$

where $\underline{H}_{\Sigma_{K_{t}}}^{g^{o}}$ is the mean curvature of the zero set of $h\left(X, K_{t}\right)$ (at least for those values of $t$ such that $K_{t}$ is scalar negative) while for $t=0$

$$
h\left(K_{1}, K_{0}=K_{1}\right)=-S_{K_{1}} .
$$

So we see an interesting link between mean curvature and the scalar curvature quantity $S_{K}$.

There are obvious results related to Theorem 1.3, For example in the situation of that theorem $\Sigma_{K_{2}}$ is itself directed almost Einstein with parallel tractor given by the part of $K_{1}$ orthogonal to $K_{2}$. This follows because from 
[33. Theoerem 4.5] the tractor connection on $\left(\Sigma_{K_{2}}, c_{K_{2}}\right)$ is a restriction of the ambient tractor connection to $K_{2}^{\perp}$. (Alternatively one may use the conformal holonomy classification [9, 50] to conclude that locally we have one of the special Einstein products discussed below whence this also follows easily.) Thus in the setting of Theorem 1.3 we have the following, for example.

Proposition 2.12. If $K_{1}$ and $K_{2}$ are spacelike and orthogonal then $\Sigma_{K_{1}} \cap$ $\Sigma_{K_{2}}$ is either empty or else is a totally umbillic hypersurface in $\left(\Sigma_{K_{2}}, c_{K_{2}}\right)$.

The reason we have taken $K_{1}$ and $K_{2}$ to be orthogonal is that otherwise $\Sigma_{K_{1}} \cap \Sigma_{K_{2}}$ may be a (double) point, collection thereof.

2.7. Tractor bundles in low dimensions. In dimensions 1 and 2 a canonical tractor connection (equivalently Cartan connection) is not determined locally by the conformal structure in the usual way. For some later constructions we use a notion of tractor connection defined on (conformally) Einstein manifolds. In dimension 2 a manifold is Einstein if and only if it has constant scalar curvature. In dimension 1 any metric is Einstein.

Let $\left(M^{d}, c\right)$ be a conformal manifold. Observe that in dimensions $d \geq 3$ if $\sigma^{-2} \boldsymbol{g}=g \in c$ is Einstein, with corresponding Einstein tractor $I$ (i.e. $\bar{I}$ is parallel and $\sigma=h(X, I)$ is nowhere vanishing), then the tractor connection is given on a section of $\mathcal{E}[1] \oplus \mathcal{E}_{a}[1] \oplus \mathcal{E}[-1]=[\mathcal{T}]_{g}$ by

$$
\nabla_{a}^{\mathcal{T}}\left(\begin{array}{c}
\alpha \\
\tau^{b} \\
\rho
\end{array}\right) \underline{\underline{g}}\left(\begin{array}{c}
\nabla_{a} \alpha-\tau_{a} \\
\nabla_{a} \tau^{b}+\delta_{a}{ }^{b}\left(\rho+\mu \sigma^{-2} \alpha\right) \\
\nabla_{a} \rho-\mu \sigma^{-2} \tau_{a}
\end{array}\right), \quad \text { or } \quad\left(\begin{array}{c}
\nabla_{a} s-t_{a} \\
\nabla_{a} t^{b}+\delta_{a}{ }^{b}(r+\mu s) \\
\nabla_{a} r-\mu t_{a}
\end{array}\right)
$$

where $\mu=-\frac{1}{2}|I|^{2}=\underline{\mathrm{Sc}}^{g} / 2 d(d-1)$ and we write $\underline{\mathrm{Sc}}^{g}$ to mean $g^{a b} \operatorname{Ric}_{a b}^{g}$ (so $J^{g} / d=\sigma^{-2} \mu$ ). On the right we have simplified the connection formula by trivialising the density bundles using the scale $\sigma$. So $s, r$ are simply functions and $t^{a}$ is an unweighted tangent field; these are given by $s=\sigma^{-1} \alpha, \sigma \tau^{a}=t^{a}$, and $r=\sigma \rho$. We will use this formula to define the tractor connection in dimensions one and two as follows. For simplicity we assume the manifold is connected. We consider the case of dimension two first. Let $(M, g)$ be a Riemannian surface where $g$ is a constant scalar curvature metric. In terms of the metric $g$ define $\mathcal{T} \stackrel{g}{=} \mathcal{E}[1] \oplus \mathcal{E}_{a}[1] \oplus \mathcal{E}[-1]$ and equip this with the connection given by the formula (14) above. In dimension one the definition is the same except that in this case we define such a connection for each $\mu \in \mathbb{R}$ and $\mu$ is not related to the Levi-Civita connection $\nabla$ or any intrinsic curvature quantity. The tractor connections so defined for manifolds of dimension 2 are (locally) flat. The same is true in dimension 1 since in this case all connections are locally flat.

Remarks: It is straightforward to see that the definition of the tractor connection above is equivalent to defining a "Schouten tensor" in dimensions 1 and 2. For the Einstein metric $g$ this is defined to be $P_{a b}^{g}:=\frac{1}{d} J^{g} \boldsymbol{g}_{a b}$ where $J^{g}:=\sigma^{-2} \mu d$. Then it is defined for other metrics in the conformal class by assuming the usual transformation formula (2). With this definition one may then decree that the tractor bundle also transforms according to the usual formula (3) and use the usual formula 5 for the tractor connection 
(now using any metric from the conformal class). That a low dimensional conformal tractor bundle is equivalent to specifying a Schouten tensor was pointed out in [14]. The point we are making here is that in dimension 2, when there is an Einstein metric in the conformal class then this leads to a preferred choice.

Next we observe that there is a uniqueness result available for the tractor connection of oriented (Riemannian signature) closed conformal 2-manifolds; in this dimension it draws on global data. By the classical uniformisation result we know that there is a conformal mapping to a metric of constant scalar curvature. On the other hand, excepting the sphere, on such surfaces if there is a constant scalar curvature metric in the conformal class then it is unique see e.g. 53] and references therein. Thus in such a conformal class (14) gives the unique tractor connection.

Using this definition of the tractor connection it is straightforward to see that the essential results from higher dimensions now extend to dimensions $d=1$ and $d=2$. Evidently we have a preferred section $X_{A}$ of $\mathcal{E}_{A}[1]$ that gives a map $\mathcal{E}^{A} \rightarrow \mathcal{E}[1]$. The formula (4) extends to give a metric $h$ in these dimensions by working in the Einstein scale (or in other conformally related scales according to the first Remark above). This metric is then preserved by the connection that (14) defines. Clearly parallel sections $I$ of the tractor bundle may again be divided into 3 classes according to whether $S_{I}:=-h(I, I)$ is positive, zero, or negative. By the same arguments as in higher dimensions (as in [33]) we then obtain the extension of Theorem 2.2 to Einstein manifolds of dimension 1 and 2. We shall exploit this in the constructions below without further mention.

Although we shall not use it explicitly here, the tractor connection in dimensions 1 and 2, as defined here, agrees with that induced from the tractor connection on higher dimensional dimensional Einstein manifolds by a suitable (local) totally umbillic embedding. For example for the unit round sphere $S^{2}$ we have $\mu=\frac{1}{2}$ and it is easily verified that the connection $\nabla^{\mathcal{T}}$ on $S^{2}$ given by (14) agrees with the tractor connection induced on $\left.N^{\perp} \subset \mathcal{T}\right|_{\Sigma}$ along an equator $\Sigma$ of the unit round $S^{3}$. Here $N$ is the normal tractor of [10, expression (12)] and writing $\mathcal{T}_{S^{3}}$ for the tractor bundle of $S^{3}, N^{\perp}$ is the subbundle of $\left.\mathcal{T}_{S^{3}}\right|_{\Sigma}$ consisting of tractors orthogonal to $N$. Similarly, by an obvious extension of the definition of $N$ to dimension 2, we then find that the $\mu=1 / 2$ connection on the unit round $S^{1}$ agrees with the tractor connection induced on $N^{\perp}$ along an equator of $S^{2}$. It follows that the tractor connection on $S^{2}$ and the $\mu=1 / 2$ tractor connection on $S^{1}$ have trivial holonomy group. In fact for dimension 1 calculations it is convenient to use the formula for the connection (also in the display 14) in terms of $s, t$ and $r$. It follows immediately from this that the equation of parallel transport in dimension 1 is equivalent to the ODE

$$
\left(s^{\prime \prime}+2 \mu s\right)^{\prime}=0 ;
$$

parallel tractors are necessarily of the form $(s, t, r)=\left(s, s^{\prime},-\left(s^{\prime \prime}+\mu s\right)\right)$ where $s$ solves the ODE. So if $\mu=1 / 2$ then we obtain $s=$ constant as well as a two dimensional family of non-constant periodic solutions. So overall a three dimensional family of solutions on $S^{1}$ as expected. On the other hand 
if we set $\mu=-\frac{1}{2}$ then an $s=1$ solution shows that $(s, t, r)=\left(1,0, \frac{1}{2}\right)$ is parallel, but since there are no periodic non-trivial solutions to $\left(s^{\prime \prime}-s\right)=0$ it follows that that there are no global parallel standard tractors on $S^{1}$ that are linearly independent of $\left(1,0, \frac{1}{2}\right)$.

2.8. Tractors on special Einstein products. The tractor bundle has rather special properties on certain product manifolds.

Definition: Let us say that $\left(M_{1}^{m_{1}} \times M_{2}^{m_{2}}, g_{1} \times g_{2}\right)$, with $m_{1}, m_{2} \geq 1$ and $m_{1}+m_{2} \geq 3$, is a special Einstein product if $\left(M_{i}, g_{i}\right), i=1,2$, is Einstein with Einstein tractor $I_{i}$ and $\left|I_{1}\right|^{2}=-\left|I_{2}\right|^{2} \neq 0$. By pull-back, we view each of the bundles $\mathcal{T}_{i}$, for $i=1,2$, as bundles on the product $M_{1} \times M_{2}$. On any Einstein manifold, with tractor bundle $\mathcal{T}$ and parallel tractor $I$ giving the Einstein structure, we shall write $\mathcal{T}^{\perp}$ to mean the orthogonal complement to $I$ in $\mathcal{T}$. Then we have (cf. [50, 9]):

Proposition 2.13. On a special Einstein product $\left(M_{1} \times M_{2}, g_{1} \times g_{2}\right)$ denote by $\left(\mathcal{T}_{i}, h_{i}, \nabla^{i}\right), i=1,2$, the respective standard tractor bundles. Let us make the definitions: $\mathcal{T}:=\mathcal{T}_{1}^{\perp} \oplus \mathcal{T}_{2}^{\perp}$ with bundle metric $h=h_{1} \times h_{2}$, where for $i=1,2, h_{i}$ is restricted to $\mathcal{T}_{i}^{\perp} ; \nabla:=\nabla^{1} \oplus \nabla^{2}$ where, for $i=1,2$, the $\nabla^{i}$ are also restricted to $\mathcal{T}_{i}{ }^{\perp}$. Then $(\mathcal{T}, h, \nabla)$ is the normal standard tractor bundle and connection. The canonical tractor $X \in \mathcal{T}[1]$ is given by $X=\left(X_{1}^{\perp}, X_{1}^{\perp}\right) \in \mathcal{T}_{1}^{\perp}[1] \oplus \mathcal{T}_{2}^{\perp}[1]$ where, for $i=1,2, X_{i}^{\perp}$ is the orthogonal projection of $X_{i}$ onto $\mathcal{T}_{i}^{\perp}[1]$.

Proof: Let $d:=m_{1}+m_{2}$. Since the $I_{i}$, for $i=1,2$, are not null, there are corresponding canonical orthogonal projections $P^{i}: \mathcal{T}_{i} \rightarrow \mathcal{T}_{i}{ }^{\perp}$ and each of these endomorphisms of $\mathcal{T}_{i}$ is parallel for $\nabla^{i}, i=1,2$. We shall use the characterisation of the normal tractor connection from [17].

By construction $\mathcal{T}$ is a vector bundle of dimension $d+2$ and the metric $h$ is preserved by the restricted product connection $\nabla$. If $I_{1}$ is spacelike $\left(\left|I_{1}\right|^{2}>0\right)$ then $I_{2}$ is timelike. Similarly if $I_{1}$ is timelike then $I_{2}$ is spacelike. In either case it is clear that $h$ has signature $(d+1,1)$. So the connection $\nabla$ is a $\mathfrak{g}$-connection where $\mathfrak{g}=s o(d+1,1)$.

Trivialise the conformal density bundles via the metric $g:=g_{1} \times g_{2}$ and view each of the canonical tractors $X_{i}, i=1,2$ as a section of $\mathcal{T}_{i}$. Now define $X:=\left(X_{1}^{\perp}, X_{2}^{\perp}\right)$ where, for $i=1,2, X_{i}^{\perp}$ is the image of $X_{i}$ under the orthogonal projection to $\mathcal{T}_{i}^{\perp}$, that is $X_{i}^{\perp}=P^{i} X_{i}$. For $i=1,2$ we have that, in the scale $g_{i}, h^{i}\left(X_{i}, I_{1}\right)=1$ and thus from $\left|I_{1}\right|^{2}=-\left|I_{2}\right|^{2}$ it follows that $X$ is null. Working in the scale $g_{1} \times g_{2}, X$ gives a map of $\mathcal{E}$ into $\mathcal{T}$ by $f \mapsto X f$. Taking $f$ here as representing a section of $\mathcal{E}[-1]$ we see that, descending to the conformal class $\left[g_{1} \times g_{2}\right], X$ determines a bundle map of $\mathcal{E}[-1]$ into $\mathcal{T}$ that we shall also denote by $X$; the latter may be viewed as a section of $\mathcal{E}[1]$. By the construction of $\mathcal{T}$, and using that $X$ is null we see that $\mathcal{T}$ has the composition structure $\mathcal{T}=\mathcal{E}[1] \in \mathcal{E}_{a}[1] \notin \mathcal{E}[-1]$. The projection $\mathcal{T} \rightarrow \mathcal{E}[1]$ is by $t \mapsto h(X, t)$ and it is easily verified that restricting $h$ to the kernel $\mathcal{T}^{(0)}=\mathcal{E}_{a}[1] \in \mathcal{E}[-1]$ of this map recovers the conformal metric $\boldsymbol{g}$ for $\left(M,\left[g_{1} \times g_{2}\right]\right)$ on $T M \cong\left(\mathcal{T}^{(0)} / \mathcal{E}[-1]\right) \otimes \mathcal{E}[1]$.

Since, for $i=1,2, \nabla^{i}$ commutes with $P^{i}$, it follows that in the scale $g_{1} \times g_{2}$ we have $\nabla X=\left(Z^{1}, Z^{2}\right)$ where the $Z^{i}$ are the Z-projectors for each $\left[\mathcal{T}_{i}\right]_{g_{i}}$ as in Section 2.1, we have used that in the given scale $P^{i} Z^{i}=Z^{i}$. It follows 
easily that the connection $\nabla$ is non-degenerate in the sense of [17] (this is the "soldering" condition).

Finally, since each of $I_{1}$ and $I_{2}$ is parallel, the curvature of $\nabla$ is the direct sum of the tractor curvatures $\Omega^{1}$ and $\Omega^{2}$ for, respectively, $\nabla^{1}$ and $\nabla^{2}$. Since $\Omega^{i} X^{i}=0, \mathrm{i}=1,2$, it follows at once that it annihilates $X$. So the curvature $\Omega$ of $\nabla$ has no torsion and the leading component of this is the Weyl curvature for $\left[g_{1} \times g_{2}\right]$. It follows from the characterisation of [17] that the connection $\nabla$ is the normal conformal standard tractor connection.

Remarks: Note that an alternative proof of the above follows easily via the cone product constructions of Fefferman-Graham (ambient) metrics in 34, Proposition 3.1] (and see also Proposition 3.7 of that article) combined with the relationship between the Fefferman-Graham metric and the conformal tractor calculus as derived in [18]. One may also obtain the result from the formula (5) by using that in the scale $g=g_{1} \times g_{2}$ the Schouten tensor $P^{g}$ is the sum of the pull backs of the Schouten tensors $P^{g_{1}}$ and $P^{g_{2}}$ from each component.

Note that in a special Einstein product the relationship between the scalar curvatures (or rather lengths of the $I_{i}$ ) is exactly that $\left(I_{1}, I_{2}\right)$ is null in $\mathcal{T}_{1} \oplus \mathcal{T}_{2}$ with respect to the product tractor metric.

\section{A (WARped) Product CONStruction of Almost Einstein MANIFOLDS}

3.1. The general product construction. It is immediate from Proposition 2.13 that special Einstein products are AE if and only if one of the factors has an additional AE structure.

Theorem 3.1. Suppose that $(M, g)=\left(M_{1} \times M_{2}, g_{1} \times g_{2}\right)$ is a special Einstein product. Then the conformal structure $(M,[g])$ admits a (directed) almost Einstein structure if and if only one of the factors $\left(M_{1},\left[g_{1}\right]\right)$ or $\left(M_{2},\left[g_{2}\right]\right)$ admits at least two linearly independent (directed) almost Einstein structures.

Proof: $I$ is a parallel non-zero standard tractor on $(M, g)$ if and only if , for $i=1,2, P^{i} I$ are each parallel and one of these is non-zero. This proves the directed case. The general result follows similarly and we leave this for the reader.

In fact the Theorem 3.1 above is the tractor statement essentially equivalent to Proposition 3.4 from [34]. Note also that in the theorem the generic case of $(M, c)$ being a (directed) almost Einstein structure will be when both of the factors admit at least two linearly independent (directed) almost Einstein structures. Since in general a parallel tractor will project into both parts $\left(\mathcal{T}_{1}^{\perp}\right.$ and $\mathcal{T}_{2}^{\perp}$ in the notation of Proposition 2.13) of the tractor bundle.

Next we observe that the scale singularity space is also easily identified.

Corollary 3.2. Suppose that $(M, g)=\left(M_{1} \times M_{2}, g_{1} \times g_{2}\right)$ is a special Einstein product. Suppose that $\left(M_{1}, g_{1}\right)$ has a linearly independent set $\left\{K_{1}, \cdots, K_{r}\right\}$ of parallel tractors which each take values in $\mathcal{T}_{1}^{\perp}$. Then, via Proposition 2.13, for each $i \in\{1, \cdots, r\}, K_{i}$ determines the almost Einstein structure $\tilde{K}_{i}:=$ $\left(K_{i}, 0\right)$ on $(M, g)$ and this has the scale singularity set $\Sigma\left(\tilde{K}_{i}\right)=\Sigma_{K_{i}} \times M_{2}$, where $\Sigma_{K_{i}} \subset M_{2}$ is the scale singularity set of $K_{i}$. 
Proof: This follows at once from Proposition 2.13 since $h\left(X, \tilde{K}_{i}\right)=\sigma_{i}$ where $\sigma_{i}$ is the pull back (via obvious map $M=M_{1} \times M_{2} \rightarrow M_{1}$ ) of $h_{1}\left(X_{1}, K_{i}\right)$ to $M$.

Remarks: In relation to the last result, note in particular that $K_{i}$ is a proper almost Einstein structure (i.e. $\Sigma_{K_{i}} \neq \emptyset$ ) if and only if $\tilde{K}_{i}$ is proper.

Also observe that assuming the existence of a parallel standard tractor $K$ which takes values in $\mathcal{T}_{1}^{\perp}$ is the same as assuming the existence of parallel tractor $K^{\prime}$ so that $\left\{K^{\prime}, I_{1}\right\}$ is a linearly independent set; given such a tractor $K^{\prime}, P^{1} K^{\prime}$ takes values in $\mathcal{T}_{1}^{\perp}$.

Evidently the Corollary 3.2 gives a general tool for constructing proper almost Einstein structures. Let us set the convention that in such products the first factor $\left(M_{1}, g_{1}\right)$ is scalar positive, that is $S_{I_{1}}>0$. For the construction of closed manifolds this way Theorem 1.2 severely limits the possibilities for $\left(M_{1}, g_{1}\right)$; to obtain a directed AE structure $M_{1}$ is necessarily a sphere.

3.2. The examples $S^{m_{1} \geq 2} \times M_{2}^{m_{2}}$. Let $\left(S^{m_{1}}, g_{1}\right)$ be the standard unit sphere of dimension $m_{1}$. If $m_{1}=2$ we fix $\mu=\frac{1}{2}$ in (14) to define the tractor connection. The sectional curvatures of this are 1, and so the parallel tractor $I_{1}$ giving $g_{1}$ has $\left|I_{1}\right|^{2}=-1$. If $\left(M_{2}^{m_{2}}, g_{2}\right)$ is any (closed) Einstein manifold with $\left|I_{2}\right|^{2}=1$ (with $I_{2}$ the parallel tractor corresponding to $g_{2}$ ) then $\operatorname{Ric}^{g_{2}}=-\left(m_{2}-1\right) g_{2}$ and

$$
(M, g)=\left(S^{m_{1}} \times M_{2}, g_{1} \times g_{2}\right)
$$

is a (closed) special Einstein product. Via Corollary 3.2 the conformal manifold $\left(M,\left[g_{1} \times g_{2}\right]\right)$ admits $m_{1}+1$ linearly independent directed AE structures $\left\{\tilde{K}_{1}, \cdots, \tilde{K}_{m+1}\right\}$. By Theorem 1.2 these are necessarily proper and scalar negative. In fact the latter point is already clear from Corollary 3.2, each non-zero parallel tractor $K_{i}$ in $\mathcal{T}_{1}^{\perp}$ has $\left|K_{i}\right|^{2}>0$ since $I_{1}$ is timelike. Thus the corresponding scale singularity set $\Sigma_{K_{i}} \subset S^{m_{1}}$ is a totally umbillic hypersphere. Then since $\left|\tilde{K}_{i}\right|^{2}=\left|K_{i}\right|^{2}$, it is clear that $\tilde{K}_{i}$ is scalar negative and its scale singularity set is the totally umbillic hypersurface $\Sigma_{\tilde{K}_{i}}=\Sigma_{K_{i}} \times M_{2}$. By Theorem 1.3 each such hypersurface $\Sigma_{\tilde{K}_{i}}$ is of constant mean curvature with respect to the scale $\sigma_{\tilde{K}_{j}}^{-2} \boldsymbol{g}$ determined by $\tilde{K}_{j}$ for $j \neq i$ in $\{1, \cdots, m+1\}$; this holds off $\Sigma_{\tilde{K}_{j}}$ and the hypersurface is minimal if $\tilde{K}_{i}$ and $\tilde{K}_{j}$ are orthogonal.

Note that if $K_{1}$ and $K_{2}$ are two linearly independent parallel tractors in $I_{1}^{\perp}$ then it may be that the corresponding scale singularity hyperspheres $\Sigma_{K_{1}}$ and $\Sigma_{K_{2}}$ intersect non-trivially. In fact generically $\Sigma_{K_{1}} \cap \Sigma_{K_{2}} \neq \emptyset$. On the other hand

$$
\Sigma_{\tilde{K}_{1}} \cap \Sigma_{\tilde{K}_{2}}=\left(\Sigma_{K_{1}} \cap \Sigma_{K_{2}}\right) \times M_{1},
$$

and so generically this is also not empty. In any case where $\Sigma_{\tilde{K}_{1}} \cap \Sigma_{\tilde{K}_{2}} \neq \emptyset$ the part $M^{\geq 0}:=M \backslash M^{-}$of $M$, where $\sigma_{1}:=h\left(X, \tilde{K}_{1}\right)$ is nonnegative (the conformal density bundle $\mathcal{E}[1]$ has a preferred positive ray subbundle $\left.\mathcal{E}_{+}[1]\right)$, is a Poincaré-Einstein manifold. On this PE manifold $M^{\geq 0}$, with $g^{o}:=\sigma_{1}^{-2} \boldsymbol{g}$ on $M^{+}:=M^{\geq 0} \backslash \Sigma_{K_{1}}$, the singularity hypersurface of $K_{2}$ (viz. $\Sigma_{K_{2}} \cap M^{\geq 0}$ ) is a totally umbillic hypersurface which meets the conformal infinity of $\left(M^{+}, g^{o}\right)$. By Proposition 2.12 if $\tilde{K}_{1}$ and $\tilde{K}_{2}$ are orthogonal then this intersection at is a totally umbillic hypersurface of the conformal infinity. 
Finally here we observe that one may be explicit about the almost Einstein structures occurring in this class of cases. Let us consider $S^{m_{1} \geq 2} \times M_{2}^{m_{2}}$ to be embedded in $\mathbb{R}^{m_{1}+1} \times M_{2}^{m_{2}}$ in the obvious way. Write $\left(x^{1}, \cdots, x^{m_{1}+1}\right)$ for the standard coordinates on the Euclidean space $\mathbb{R}^{m_{1}+1}$. Then away from $x^{1}=0$ on $S^{m_{1} \geq 2} \times M_{2}^{m_{2}}$ a typical metric in the class here is

$$
g^{o}=\left(x^{1}\right)^{-2}\left(g_{\mathrm{rd}} \times g_{2}\right) .
$$

Up to a conformal symmetry all the AE structures in this class are like this.

3.3. The regular products $S^{1} \times M_{2}^{m_{2}}$ and periodic AE structures. Since $S^{1}$ is 1-dimensional there is a choice involved in the definition of the tractor connection. On the standard unit circle let us term the connection (14) with $\mu=\frac{1}{2}$ to be the regular tractor connection. Using this the scale tractor $I_{1}$ on $S^{1}$ is parallel and has $\left|I_{1}\right|^{2}=-1$. With reference to the ODE (15), the parallel tractors on $S^{1}$ orthogonal to $I_{1}$ are the solutions of $s^{\prime \prime}+s=0$ and so, taking the usual parametrisation, have two scale singularity points. Thus, as for the cases of $m_{1} \geq 2$ above, if $\left(M_{2}^{m_{2}}, g_{2}\right)$ is any (closed) Einstein manifold with Ric ${ }^{g_{2}}=-\left(m_{2}-1\right) g_{2}$ then $(M, g)=\left(S^{1} \times M_{2}, g_{1} \times g_{2}\right)$ is a (closed) special Einstein product with a $\left(m_{1}+1=2\right)$-dimensional space of almost Einstein structures compatible with the conformal structure $\left[g_{1} \times\right.$ $\left.g_{2}\right]$. Everything works as in the case of $m_{1} \geq 2$ above except that for two linearly independent parallel tractors $K_{1}$ and $K_{2}$ on $S^{1}$ the corresponding scale singularity sets, respectively, $\Sigma_{K_{1}}$ and $\Sigma_{K_{2}}$ do not intersect unless they coincide. This has the obvious implication for the scale singularity sets, $\Sigma_{\tilde{K}_{1}}=\Sigma_{K_{1}} \times M_{1}$ and $\Sigma_{\tilde{K}_{2}}=\Sigma_{K_{2}} \times M_{1}$, of the parallel tractors $\tilde{K}_{1}$ and $\tilde{K}_{2}$ on $(M, c)$.

Since the connections of (14) are natural for the given Riemannian Einstein structure we may clearly pull these back to any covering space. Thus for $K$ a parallel tractor on $S^{1}$ its pull back (which we shall also denote by $K)$ is a parallel tractor, and so a directed almost Einstein structure, on any covering space of $S^{1}$. It is straightforward to give a positive length parallel tractor $K$ on $S^{1}$ with two scale singularity points. Then on a $k$-fold covering $\tilde{S}^{1}$ of $S^{1}$ the singularity set of $K$ consists of $2 k$ isolated points. The corresponding special Einstein product $(M, g)=\left(M_{1} \times \tilde{S}^{1}, g_{1} \times g_{2}\right)$ (with $\left(M_{1}^{m_{2}}, g_{1}\right)$ as above) is a periodic almost Einstein manifold with $2 k$ scale singularity points. This applies with $k$ infinite and $\tilde{S}^{1}$ is then $\mathbb{R}$. For finite $k$ the $2 k$ singularity points may be thought of as zeros of periodic solutions of (15) with appropriate $\mu$ in relation to the range of the independent variable.

3.4. "Exotic" $M_{1}^{m_{1}} \times S^{1}$ special Einstein products. If we equip the usual unit circle $\left(S^{1}, g_{2}\right)$ with the tractor connection (14) with $\mu=-\frac{1}{2}$ then the globally parallel tractor $I_{2}: \stackrel{g_{2}}{=}\left(\sigma, 0, \frac{1}{2} \sigma^{-1}\right)$ has $\left|I_{2}\right|^{2}=1$, and so in this sense $S^{1}$ behaves as a negative scalar curvature Einstein manifold. (In this sense these cases are essentially a special case of those in Section 3.2 above. However some special comments are in order.) In particular we may form special Einstein products

$$
(M, g):=\left(M_{1}^{m_{1}} \times S^{1}, g_{1} \times g_{2}\right)
$$

where now $\operatorname{Ric}^{g_{1}}=\left(m_{1}-1\right) g_{1} ; g_{1}$ has positive scalar curvature. 
There are no non-trivial parallel tractors on $S^{1}$ in $I_{2}^{\perp}$ and so if $M_{1}$ is closed and Riemannian then it follows easily (using also Theorem 1.2) that $(M,[g])$ admits compatible directed AE structures if and only if $\left(M_{1}, g_{1}\right)$ is isometric to the unit round sphere. Thus the only closed manifolds admitting directed almost Einstein structures obtained in this way are the unit sphere products as follows. (This shows we are really reduced to a special case of the examples from Section 3.2.)

Proposition 3.3. The product of unit spheres

$$
S^{n \geq 2} \times S^{1}
$$

with its usual metric and conformal structure admits an $(n+1)$-dimensional vector space of parallel tractors, equivalently a $(n+1)$-dimensional family of directed $A E$ structures. The product of unit circles $S^{1} \times S^{1}$ admits a 4-dimensional family of directed AE structures compatible with its given conformal structure.

Note that by the uniqueness (up to isomorphism) of the normal tractor bundle it follows that the tractor bundle on the product here is necessarily given by Theorem 2.13 , with $S^{1}$ taken with the $\mu=-\frac{1}{2}$ tractor connection (as in (14)). The result for $S^{1} \times S^{1}$ follows as we may choose which factor carries the positive length Einstein tractor.

3.5. The examples $\mathbb{R P}_{m_{1} \geq 1} \times M_{2}^{m_{2}}$. Recall that the standard conformal structure $c$ on $\mathbb{R P}_{m_{1} \geq 1}$ admits an Einstein tractor $I_{1}$, with $\left|I_{1}\right|^{2}=-1$ and in $I_{1}^{\perp}$ an $\left(m_{1}+1\right)$-dimensional family of undirected almost Einstein structures. We write $g_{1}$ for the Einstein metric corresponding to $I_{1}$. We may form products as for the cases above: If $\left(M_{2}^{m_{2}}, g_{2}\right)$ is any (closed) Einstein manifold with $\operatorname{Ric}^{g_{2}}=-\left(m_{2}-1\right) g_{2}$ then

$$
(M, g)=\left(\mathbb{R P}_{m_{1}} \times M_{2}, g_{1} \times g_{2}\right)
$$

is a (closed) special Einstein product. Let us assume that $M_{2}$ is connected. Since for each parallel tractor class $[K]$, with locally $[K] \ni K^{\prime} \in I_{1}^{\perp}$, and with singularity hypersurface $\Sigma_{[K]}$, the "bulk" $\mathbb{R P}_{m_{1}} \backslash \Sigma_{[K]}$ is connected and so it follows that $M \backslash \Sigma_{[\tilde{K}]}$ is also connected, as recall $\Sigma_{[\tilde{K}]}=\Sigma_{[K]} \times M_{2}$ (here locally $\tilde{K}=\left(K^{\prime}, 0\right)$ as in Corollary $[3.2)$. Thus $[\tilde{K}]$ is undirected.

\section{Poincaré-Einstein subproducts and Compactifications}

4.1. Decomposable conformal holonomy. In this section we discuss scalar negative almost Einstein spaces which have decomposable conformal holonomy. This discussion will allow us to determine which of the PoincaréEinstein collars, as introduced in [34, are suitable for compactification.

First we recall the notion of a metric cone. Let $\left(M^{d}, g\right)$ be a Riemannian space of dimension $d$. Then we define $\hat{M}:=M \times \mathbb{R}_{+}$. The cone metric of $g$ with timelike Euler vector $t \cdot \partial_{t}$ is defined on $\hat{M}$ by

$$
\hat{g}:=-d t^{2}+t^{2} \cdot g,
$$

where $t$ is the coordinate of $\mathbb{R}_{+}$. We call $\hat{g}$ the timelike cone metric of $g$. In 34] we have shown that any metric of the form

$$
g=r^{-2}\left(d r^{2}+\left(1-\mu r^{2} / 2\right)^{2} g_{1}+\left(1+\mu r^{2} / 2\right)^{2} g_{2}\right),
$$


where $\mu>0$ and $g_{1}, g_{2}$ are Einstein metrics of dimension $m_{1} \geq 1$, resp., $m_{2} \geq 1$, with $2 m_{1}\left(m_{1}-1\right) \mu:=\operatorname{Sc}\left(g_{1}\right)$ and $2 m_{2}\left(m_{2}-1\right) \mu:=-\operatorname{Sc}\left(g_{2}\right)$, has the property that its timelike cone $\hat{g}=-d t^{2}+t^{2} \cdot g$ is decomposable as a Riemannian manifold. Note that with the coordinate change $s=\ln \left(\sqrt{\frac{\mu}{2}} \cdot r\right)$ for $r>0$ the metric $g$ takes the form

$$
d s^{2}+2 \mu\left(\sinh ^{2}(s) \cdot g_{1}+\cosh ^{2}(s) \cdot g_{2}\right) .
$$

In the case that the Poincaré-Einstein collar metric (16) has $g_{1}$ a round sphere metric then it is easily seen directly, or via the examples of section 3.2, that we may compactify the collar. So our focus here is to determine whether there are other cases where compactification is possible.

Spaces with a decomposable cone have been discussed earlier in, for example, [28] and [48, 50]. In general, one can show that any metric with a decomposable timelike metric cone is (at least on some open dense subset) locally isometric to a metric of the form (16). In fact, if the base metric is complete then the following result is true.

Theorem 4.1. [3] Let $(M, g)$ be a complete Riemannian manifold of dimension at least two and such that its timelike cone $\hat{M}$, with cone metric $\hat{g}:=-d t^{2}+t^{2} g$, has decomposable holonomy algebra. Then, on some open dense subset and for each connected component thereof, $g$ is isometric to a metric of the form

$$
r^{-2}\left(d r^{2}+\left(1-\mu r^{2} / 2\right)^{2} g_{1}+\left(1+\mu r^{2} / 2\right)^{2} g_{2}\right),
$$

with $\mu>0$, where the $g_{2}$ term is possibly absent, and where $g_{1}$ has positive constant sectional curvature.

We shall also need the notion of conformal holonomy, which we now recall. Let $\left(M^{d}, c\right)$ be a conformal structure of dimension $d \geq 3$. On $\left(M^{d}, c\right)$ we have the tractor bundle $(\mathcal{T}, \nabla)$ with its canonical normal connection. As for any vector bundle connection, the tractor connection $\nabla$ on $\mathcal{T}$ has a uniquely defined holonomy algebra and group (cf. [43]). In the following, we denote the holonomy group of $(\mathcal{T}, \nabla)$ on $\left(M^{d}, c\right)$ by $\operatorname{Hol}(\mathcal{T})$. We shall call $\operatorname{Hol}(\mathcal{T})$ the conformal holonomy group of $\left(M^{d}, c\right)$ (cf. $\left.[9,48,50]\right)$. The corresponding conformal holonomy algebra is denoted by $\mathfrak{h o l}(\mathcal{T})$. The conformal holonomy group is a subgroup of the Möbius group $O(d+1,1)$, whose standard representation space is $\mathbb{R}^{d+1,1}$. If the restriction of the standard representation of $\operatorname{Hol}(\mathcal{T})$ on $\mathbb{R}^{d+1,1}$ decomposes into a direct sum, then we call the conformal holonomy group decomposable. Accordingly, if the conformal holonomy algebra $\mathfrak{h o r}(\mathcal{T})$ splits into a direct sum, then we call $\mathfrak{h o l}(\mathcal{T})$ decomposable. Note that for simply connected spaces $\left(M^{d}, c\right)$ decomposability of $\mathfrak{h o l}(\mathcal{T})$ and $\operatorname{Hol}(\mathcal{T})$ are equivalent.

Furthermore, note that if the conformal structure $c$ on $M^{d}$ includes an analytic metric $g$, then the tractor connection $\nabla$ is analytic. This implies that the conformal holonomy algebra $\mathfrak{h o r}(T)$ of $(M, c)$ is equal to the conformal holonomy algebra of any open subspace $U$ of $(M, c)$. Here we mean $U$ is equipped with the restricted conformal structure. It is also a fact that the conformal holonomy group and the holonomy group of the timelike cone over any Einstein space of scalar curvature $-d(d-1)$ coincide (see [34]). 
This shows that any metric of the form (16) has decomposable conformal holonomy group.

Now let us assume that $\left(M^{d}, c\right)$ with $\operatorname{dim}(M)=d \geq 3$ is a compact conformal space, which admits an almost Einstein structure $I$ which is scalar negative (that is $S_{I}<0$ ), and whose conformal holonomy algebra $\mathfrak{h o l}(\mathcal{T})$ decomposes as a direct sum $\mathfrak{a} \times \mathfrak{b}$ on $\mathbb{R}^{d+1,1}$. Then we can describe the local geometry of $c$ almost everywhere as follows.

Theorem 4.2. Let $\left(M^{d}, c, I\right)$ be either a Poincaré-Einstein manifold or a closed scalar negative directed AE structure. Suppose further that $\left(M^{d}, c\right)$ has a decomposable conformal holonomy algebra $\mathfrak{h o l}(\mathcal{T})$. Then, on an open dense subset of $M$, the conformal class c locally includes a metric of the form (16), where $g_{1}$ has positive constant sectional curvature.

Proof. Let $I$ denote the given almost Einstein structure with $S_{I}<0$; replacing $I$ by a constant multiple if necessary we may assume $S_{I}=-1$. We denote by $M^{o}$ the complement of the scale singularity set $\Sigma_{I}$, of $I$ in $M$. Writing $\sigma:=h(X, I)$, then, in the restriction of $c$ to $M^{o}, g^{o}=\sigma^{-2} \boldsymbol{g}$ is complete Einstein metric of negative scalar curvature $-d(d-1)$. We denote by $(\tilde{M}, \tilde{g})$ the universal covering space of $\left(M^{o}, g^{o}\right)$. Since, as a subset of $M$, the space $\left(M^{o}, g^{o}\right)$ has a decomposable conformal holonomy algebra, the conformal holonomy group of the universal covering space $(\tilde{M}, \tilde{g})$ is decomposable. Hence we can conclude here that the timelike cone $(\hat{M}, \hat{g})$ over $(\tilde{M}, \tilde{g})$ decomposes as a Riemannian manifold. And since $(\tilde{M}, \tilde{g})$ is complete, we see via Theorem 4.1 that the universal covering space takes locally (on an open dense subset) the stated geometric form. But then it is also clear that $\left(M^{o}, g^{o}\right)$ is locally isometric (on an open dense subset) to a metric of the form (16), where $g_{1}$ has positive constant sectional curvature.

Theorem 4.2 has some immediate consequences.

Corollary 4.3. Let $\left(M^{d}, c\right)$ be a closed directed scalar negative AE space (resp. a compact Poincaré-Einstein space) with decomposable conformal holonomy algebra and with conformal infinity $\left(\Sigma, c_{\Sigma}\right)$. Then, on an open dense subspace, $\left(\Sigma, c_{\Sigma}\right)$ is locally conformally equivalent to a product $S^{m_{1}} \times N$, where $S^{m_{1}}$ denotes the unit sphere of dimension $m_{1}$, and $N$ is a space of dimension $m_{2}=d-m_{1}-1$ equipped with an Einstein metric $h$ of negative scalar curvature $-m_{2}\left(m_{2}-1\right)$. Then, on an open dense subset of $M$, the space $\left(M^{d}, c\right)$ is locally equivalent to the conformal structure of $S^{m_{1}+1} \times N$ with metric $g_{r d} \times h$.

Proof. Let us consider the conformal structure $c$ locally on a (small) collar of the conformal infinity $\left(\Sigma, c_{\Sigma}\right)$. By Theorem 4.2 we know that the Poincaré-Einstein metric $g^{o}$, in the conformal class $c$, on such a collar is almost everywhere given by a metric of the form

$$
r^{-2}\left(d r^{2}+\left(1-r^{2} / 4\right)^{2} g_{r d}+\left(1+r^{2} / 4\right)^{2} h\right),
$$

where $g_{r d}$ is the standard unit sphere metric (i.e. with constant sectional curvature 1 and $\mu=1 / 2$ ). The displayed metric is conformally equivalent to a metric $g_{r d} \times \tilde{h}$. Here the coordinate $r$ is defined on some half interval $[0, \varepsilon)$. This form of the Poincaré-Einstein metric also shows that the (local) 
boundary conformal structure on $\Sigma$ is, on an open dense subspace, given by $g_{r d} \times h$, where $g_{r d}$ is locally isometric to the round unit sphere in dimension $m_{1}$.

Corollary 4.4. Let $M^{m_{1}} \times N^{m_{2}}\left(m_{1} \geq 2, m_{2} \geq 0\right)$ with metric $g=g_{1} \times g_{2}$ be either Einstein with $m_{2}=0$ or a special Einstein product. Assume that, in either case, $\left(M, g_{1}\right)$ has positive scalar curvature, but its sectional curvature is non-constant. Then the Poincaré-Einstein collar of [34] for $g_{1} \times g_{2}$ cannot be compactified. That is, there is no conformal embedding of that collar into a compact Poincaré-Einstein space.

Proof. Let us assume that there exists a collar equipped with a PoincaréEinstein metric of the form

$$
r^{-2}\left(d r^{2}+\left(1-r^{2} / 4\right)^{2} g_{1}+\left(1+r^{2} / 4\right)^{2} g_{2}\right),
$$

where $g_{1}$ has non-constant sectional curvature, which can be embedded into some compact Poincaré-Einstein space $(P, h)$ (whose conformal infinity contains the boundary of that collar as a subspace). Now, the conformal holonomy algebra of that collar is decomposable and it follows, from the analyticity of the Einstein interior, that this coincides with the conformal holonomy algebra of $(P, h)$. Thus, from the previous Corollary, on an open dense subspace, the bulk of $(P, h)$ is locally isometric to a metric of the form (16), with $g_{1}$ a piece of the round sphere. This contradicts the assumption on the embedded collar.

Note that, in particular, Corollary 4.4 states that the Poincaré-Einstein metric

$$
\frac{1}{r^{2}}\left(d r^{2}+\left(1-\mu \cdot r^{2} / 2\right) \cdot g_{1}\right)
$$

on some collar $[0, \varepsilon) \times M$ for an Einstein space $\left(M^{n}, g_{1}\right)$ of dimension $n$ with positive scalar curvature cannot be compactified, unless $\left(M, g_{1}\right)$ is conformally flat. In particular, if $M$ is closed and simply connected then $\left(M, g_{1}\right)$ has to be (up to a constant scale) the round unit sphere $S^{n}$.

4.2. A global statement. For each special Einstein product $g_{1} \times g_{2}$ Theorem 4.1 of [34] gives a Poincaré-Einstein collar. Using the relationship between the conformal tractor connection and the ambient metric, it is straightforward to see that by construction, the Poincaré-Einstein collar, so obtained, has the same conformal holonomy group as its boundary. Both holonomy groups are decomposable. In fact, it is also straightforward to see that this equality of conformal holonomy groups of a boundary and its bulk characterises, in the decomposable case, the Poincaré-Einstein collar metric of 34 uniquely.

The following result states that Example 3.2 is the basic construction principle for closed almost Einstein spaces, whose collar at the singularity set is equivalent to the collar metric as introduced in [34.

Theorem 4.5. Assume that the space $\left(\Sigma, c_{\Sigma}\right)$ is conformal to a special Einstein product $A^{r} \times B^{s}$ of dimension $r+s=d-1$ with metric $g_{1} \times g_{2}$, where $S c\left(g_{1}\right)=r(r-1)$. If $\left(M^{d}, c, I\right)$ is a simply connected, closed, and directed $A E$ space with conformal infinity $\left(\Sigma, c_{\Sigma}\right)$ such that the conformal holonomy 
groups of $\left(\Sigma, c_{\Sigma}\right)$ and $\left(M^{n}, c\right)$ coincide, then $\left(A, g_{1}\right)$ is isometric to $\left(S^{r}, g_{r d}\right)$ and $\left(M^{d}, c\right)$ is conformal to $S^{r+1} \times B$ with the metric $g_{r d} \times g_{2}$.

Proof. The volume form $\alpha:=\operatorname{vol}\left(g_{2}\right)$ for $g_{2}$ on $B$ can be considered as a parallel $s$-form on $A \times B$ with metric $g_{1} \times g_{2}$. This also means that $\alpha$ is a normalised conformal Killing $s$-form on $\left(\Sigma, c_{\Sigma}\right)$ in the sense of [50, 48], i.e., the corresponding $(s+1)$-form tractor $K(\alpha)$ is simple and parallel with respect to the tractor connection $\nabla$ on $\Sigma$. The existence of this $(s+1)$ form tractor implies the decomposability of the conformal holonomy group $\operatorname{Hol}(\Sigma, \mathcal{T})$. Since, by assumption, $\operatorname{Hol}(\Sigma, \mathcal{T})=\operatorname{Hol}(M, \mathcal{T})$ and $M$ is simply connected, it follows that there exists a parallel and simple $(s+1)$-form tractor $T$ on $(M, c)$, whose restriction to $\mathcal{T}$ on $\Sigma$ (which is in a natural and unique way a subbundle of $\mathcal{T}$ on $M$, see [33, Theorem 4.5]) gives rise to $K(\alpha)$. We denote by $\beta$ the normalised conformal Killing $s$-form on $\left(M^{n}, c\right)$, which corresponds to $T$. Then, by construction, the pull back of the $s$-form $\beta$, by the embedding $\iota: \Sigma \rightarrow M$, gives the $s$-form $\alpha$.

We want to argue now that the $s$-form $\beta$ has no zeros. This is clear on the singularity set $\Sigma$, since $\iota^{*} \beta=\alpha$ has no zeros. So let us consider $\beta$ on the bulk $M^{o}$ of $M$ with Poincaré-Einstein metric $g^{o}$ and corresponding almost Einstein structure $I$. Recall that the product of a real line with the timelike cone over $\left(M^{o}, g^{o}\right)$ gives rise to the Fefferman-Graham ambient metric of $\left(M^{o}, g^{o}\right)$ [50, 34]. Since, by construction, $\left.I\right\lrcorner T=0$, it is clear that the $(s+1)$-form tractor $T$ corresponds, via the Fefferman-Graham ambient metric, in a unique way to a parallel and simple $(s+1)$-form $\hat{T}$ on the timelike cone $\hat{M}$ over the bulk $\left(M^{o}, g^{o}\right)$. This $(s+1)$-form $\hat{T}$ has, by construction, negative length, i.e., it can be expressed by a wedge product of one timelike 1 -form and some simple spacelike $s$-form. (Note that the $(s+1)$-form $\hat{T}$ can be interpreted as the volume form of the timelike cone over $\left(B, g_{2}\right)$.) This shows that inserting the Euler vector $t \cdot \partial_{t}$ into $\hat{T}$ is nowhere vanishing. And, since the pull back of the $s$-form $\left.\left(t \cdot \partial_{t}\right)\right\lrcorner \hat{T}$ via the embedding of $M^{o}$ (as the 1-level) into the timelike cone gives rise to $\beta$, we can conclude that $\beta$ has no zeros on $M^{o}$.

Now we can choose a metric $h$ in $c$ such that $\beta$ has constant length on $M$. In this scale $\beta$ is a parallel $s$-form (cf. [50]). Since $(M, h)$ is simply connected and closed, this proves that $(M, c)$ is conformal to a special Einstein product $C \times D$ with metric $h_{1} \times h_{2}$, where $S c\left(h_{1}\right)>0$. However, from Corollary 4.3 we know that $g_{1}$ and $h_{1}$ have positive constant sectional curvature. Moreover, since $C$ is closed and simply connected it has to be the round sphere $S^{r+1}$. And, since $\hat{T}$ can be interpreted as the volume form of the timelike cone over $\left(B, g_{2}\right)$, we conclude that $\left(D, h_{2}\right)$ is isometric to $\left(B, g_{2}\right)$. Finally, since $\left.I\right\lrcorner T=0$, we see that $I$ on $M$ comes from an almost Einstein structure on the factor $S^{r+1}$. This shows that the boundary space $\Sigma$ is $S^{r} \times B$ with metric $g_{1} \times g_{2}$, where $g_{1}$ is the standard sphere metric.

\section{REFERENCES}

[1] P. Albin, Renormalizing Curvature Integrals on Poincare-Einstein Manifolds, math.DG/0504161 
[2] D. Alekseevski, Groups of conformal transformations of Riemannian spaces, Math. USSR Sbornik, 18 (1972), 285-301.

[3] D. Alekseevski, V. Cortés, A. Galaev, and T. Leistner, Cones over pseudoRiemannian manifolds and their holonomy, arXiv:0707.3063

[4] S. Alexakis, and R. Mazzeo, Renormalized area and properly embedded minimal surfaces in hyperbolic 3-manifolds, arXiv:0802.2250

[5] M. Anderson, $L^{2}$ curvature and volume renormalization of AHE metrics on 4manifolds, Math. Res. Lett. 8 (2001), no. 1-2, 171-188.

[6] M.T. Anderson, Boundary regularity, uniqueness and non-uniqueness for AH Einstein metrics on 4-manifolds, Adv. Math. 179 (2003), 205-249.

[7] M.T. Anderson, Geometric aspects of the AdS/CFT correspondence, in: AdS/CFT correspondence: Einstein metrics and their conformal boundaries, 1-31, IRMA Lect. Math. Theor. Phys., 8, Eur. Math. Soc., Zürich, 2005.

[8] M.T. Anderson, Einstein metrics with prescribed conformal infinity on 4-manifolds, GAFA, to appear, arXiv:math/0105243

[9] S. Armstrong, Definite signature conformal holonomy: a complete classification, J. Geom. Phys. 57 (2007), 2024-2048.

[10] T.N. Bailey, M.G. Eastwood, and A.R. Gover, Thomas's structure bundle for conformal, projective and related structures, Rocky Mountain J. Math. 24 (1994), 11911217.

[11] A.L. Besse, Einstein manifolds, Ergebnisse der Mathematik und ihrer Grenzgebiete (3), 10, Springer-Verlag, Berlin, xii+510pp, 1987.

[12] O. Biquard, Métriques d'Einstein asymptotiquement symétriques, Astérisque, No. 265 (2000), vi+109 pp.

[13] H.W. Brinkmann, Einstein spaces which are mapped conformally on each other. Math. Ann. 94, (1925) 119-145.

[14] D. Calderbank, Möbius structures and two-dimensional Einstein-Weyl geometry, J. Reine Angew. Math. 504 (1998), 37-53.

[15] A. Čap, Infinitesimal Automorphisms and Deformations of Parabolic Geometries, J. Eur. Math. Soc. 10, (2008) 415-437.

[16] A. Čap, and A.R. Gover, Tractor bundles for irreducible parabolic geometries, Global analysis and harmonic analysis (Marseille-Luminy, 1999), 129-154, Sémin. Congr., 4, Soc. Math. France, Paris, 2000.

[17] A. Čap, and A.R. Gover, Tractor calculi for parabolic geometries, Trans. Amer. Math. Soc. 354 (2002), 1511-1548.

[18] A. Čap, and A.R. Gover, Standard tractors and the conformal ambient metric construction, Ann. Global Anal. Geom. 24 (2003), 231-295.

[19] A. Čap, and A.R. Gover, CR-Tractors and the Fefferman Space, arXiv:math/0611938

[20] A. Chang, J. Qing, and P. Yang, On the renormalized volumes for conformally compact Einstein manifolds, arXiv:math/0512376

[21] A. Derdzinski, and G. Maschler, Local classification of conformally-Einstein Kähler metrics in higher dimensions, Proc. London Math. Soc. (3) 87 (2003), 779-819.

[22] A. Derdzinski, and G. Maschler, Special Kähler-Ricci potentials on compact Kähler manifolds, J. Reine Angew. Math., 593 (2006), 73-116.

[23] Eastwood, M.G., Notes on conformal differential geometry, Supp. Rend. Circ. Matem. Palermo, Ser. II, Suppl., 43, (1996), 57-76.

[24] C. Fefferman, and C.R. Graham, Conformal invariants in: The mathematical heritage of Élie Cartan (Lyon, 1984). Astérisque 1985, Numero Hors Serie, 95-116.

[25] C. Fefferman, and C.R. Graham, Q-curvature and Poincaré metrics, Math. Res. Lett. 9 (2002), 139-151.

[26] C. Fefferman, and C.R. Graham, The Ambient Metric, arXiv:0710.0919

[27] J. Ferrand, The action of conformal transformations on a Riemannian manifold, Math. Ann., 304 (1996), 277-291.

[28] S. Gallot, Équations différentielles caractéristiques de la sphère, Ann. Sci. École Norm. Sup. (4) 12 (1979), 235-267. 
[29] A.R. Gover, Aspects of parabolic invariant theory, Rend. Circ. Mat. Palermo (2) Suppl. No. 59 (1999), 25-47.

[30] A.R. Gover, Almost conformally Einstein manifolds and obstructions, in Differential geometry and its applications, 247-260, Matfyzpress, Prague, 2005. Electronic: arXiv:math/0412393

[31] A.R. Gover, Laplacian operators and Q-curvature on conformally Einstein manifolds, Math. Ann. 336 (2006), 311-334.

[32] A.R. Gover, Conformal Dirichlet-Neumann Maps and Poincaré-Einstein Manifolds, SIGMA Symmetry Integrability Geom. Methods Appl. 3 (2007), Paper 100, 21 pp.

[33] A.R. Gover, Almost Einstein and Poincaré-Einstein manifolds in Riemannian signature, arXiv:0803.3510

[34] A.R. Gover, and F. Leitner, A sub-product construction of Poincare-Einstein metrics, arXiv:math/0608044

[35] A.R. Gover, and L. Peterson, Conformally invariant powers of the Laplacian, Qcurvature, and tractor calculus Comm. Math. Phys. 235 (2003), 339-378.

[36] A.R. Gover, and J. Šilhan, The conformal Killing equation on forms - prolongations and applications, Differential Geometry and Its Applications, 26, 244-266. arXiv:math/0601751

[37] C.R. Graham, and K. Hirachi, The ambient obstruction tensor and Q-curvature, AdS/CFT correspondence: Einstein metrics and their conformal boundaries, 59-71, IRMA Lect. Math. Theor. Phys., 8, Eur. Math. Soc., Zrich, 2005.

[38] C.R. Graham, and A. Juhl, Holographic formula for Q-curvature, Adv. Math. 216 (2007), 841-853.

[39] C.R. Graham, and J.M. Lee, Einstein metrics with prescribed conformal infinity on the ball, Adv. Math. 87 (1991), 186-225.

[40] C.R. Graham, and E. Witten, Conformal anomaly of submanifold observables in AdS/CFT correspondence, Nuclear Phys. B 546 (1999), no. 1-2, 52-64.

[41] C.R. Graham, and M. Zworski, Scattering matrix in conformal geometry, Invent. Math. 152 (2003), 89-118.

[42] C. Guillarmou, Generalized Krein formula and determinants for even dimensional Poincare-Einstein manifolds, arXiv:math/0512173.

[43] S. Kobayashi and K. Nomizu, Foundations of differential geometry, Vol I, Interscience Publishers, a division of John Wiley \& Sons, New York-Lond on 1963 xi+329 pp.

[44] W. Kühnel and H.-B. Rademacher, Essential conformal fields in pseudo-Riemannian geometry, J. Math. Pures Appl. (9) 74 (1995), 453-481.

[45] W. Kühnel and H.-B. Rademacher, Essential conformal vector fields in pseudoRiemannian geometry. II, J. Math. Sci. Univ. Tokyo, 4 (1997), 649-662.

[46] J.M. Lee, Fredholm operators and Einstein metrics on conformally compact manifolds, Mem. Amer. Math. Soc. 183 (2006), vi+83 pp.

[47] J.M. Lee, The spectrum of an asymptotically hyperbolic Einstein manifold, Comm. Anal. Geom. 3 (1995), 253-271.

[48] F. Leitner, Conformal Killing forms with normalisation condition, Rend. Circ. Mat. Palermo (2) Suppl. No. 75 (2005), 279-292, math.DG/0406316.

[49] F. Leitner, Leitner, Felipe On transversally symmetric pseudo-Einstein and Fefferman-Einstein spaces, Math. Z. 256 (2007), 443-459.

[50] F. Leitner, Normal Conformal Killing Forms, arXiv:math/0406316

[51] J. Maldacena The large $N$ limit of superconformal field theories and supergravity, Adv. Theor. Math. Phys. 2 (1998), 231-252.

[52] R. Mazzeo, and F. Pacard, Maskit combinations of Poincaré-Einstein metrics, Adv. Math. 204 (2006), 379-412.

[53] R. Mazzeo, and M. Taylor, Curvature and uniformization, Israel J. Math. 130 (2002), 323-346.

[54] B. O'Neill, Semi-Riemannian geometry. With applications to relativity. Pure and Applied Mathematics, 103. Academic Press, Inc., New York, 1983.

[55] T.Y. Thomas, On conformal geometry, Proc. Natl. Acad. Sci. USA 12, 352-359 (1926) 
[56] X. Wang, On conformally compact Einstein manifolds, Math. Res. Lett. 8 (2001), 671-688.

[57] E. Witten Anti de Sitter space and holography, Adv. Theor. Math. Phys. 2 (1998), 253-291.

[58] Y. Yoshimatsu, On a theorem of Alekseevskii concerning conformal transformations, J. Math. Soc. Japan, 28 (1976), 278-289.

Department of Mathematics, The University of Auckland, Private Bag 92019, Auckland 1, New Zealand

E-mail address: gover@math.auckland.ac.nz

Universitat Stuttgart, Institut fr Geometrie und Topologie, Mathematics Department, Pfaffenwaldring 57, D-70550 Stuttgart

E-mail address: leitner@mathematik.uni-stuttgart.de 